\title{
From Generalized Dirac Equations to a Candidate for Dark Energy
}

\author{
U. D. Jentschura and B. J. Wundt \\ Department of Physics, Missouri University of Science and Technology, Rolla, MO 65409, USA \\ Correspondence should be addressed to U. D. Jentschura; jentschurau@mst.edu
}

Received 12 November 2012; Accepted 1 December 2012

Academic Editors: G. A. Alves, C. A. D. S. Pires, and F.-H. Liu

Copyright (c) 2013 U. D. Jentschura and B. J. Wundt. This is an open access article distributed under the Creative Commons Attribution License, which permits unrestricted use, distribution, and reproduction in any medium, provided the original work is properly cited.

\begin{abstract}
We consider extensions of the Dirac equation with mass terms $m_{1}+\mathrm{i} \gamma^{5} m_{2}$ and $\mathrm{i} m_{1}+\gamma^{5} m_{2}$. The corresponding Hamiltonians are Hermitian and pseudo-Hermitian $\left(\gamma^{5}\right.$ Hermitian), respectively. The fundamental spinor solutions for all generalized Dirac equations are found in the helicity basis and brought into concise analytic form. We postulate that the time-ordered product of field operators should yield the Feynman propagator (i $\epsilon$ prescription), and we also postulate that the tardyonic as well as tachyonic Dirac equations should have a smooth massless limit. These postulates lead to sum rules that connect the form of the fundamental field anticommutators with the tensor sums of the fundamental plane-wave eigenspinors and the projectors over positive-energy and negative-energy states. In the massless case, the sum rules are fulfilled by two egregiously simple, distinguished functional forms. The first sum rule remains valid in the case of a tardyonic theory and leads to the canonical massive Dirac field. The second sum rule is valid for a tachyonic mass term and leads to a natural suppression of the right-handed helicity states for tachyonic particles and left-handed helicity states for tachyonic spin-1/2 antiparticles. When applied to neutrinos, the theory contains a free tachyonic mass parameter. Tachyons are known to be repulsed by gravity. We discuss a possible role of a tachyonic neutrino as a contribution to the accelerated expansion of the Universe "dark energy."
\end{abstract}

\section{Introduction}

1.1. Generalized Dirac Equations: Mass Terms and Dispersion Relations. Dirac is often quoted saying in some of his talks that the equation that carries his name $[1,2]$ is "more intelligent than its inventor." Of course, it needs to be added that it was Dirac himself who found most of the additional insight. Here, we are concerned with extensions of the Dirac equation which contain both tardyonic and tachyonic mass terms. Tardyonic (subluminal) mass terms lead to dispersion relations of the form $E=\sqrt{\vec{p}^{2}+m^{2}}$, whereas tachyonic mass terms lead to superluminal dispersion relations of the form $E=\sqrt{\vec{p}^{2}-m^{2}}$, where $E$ is the energy and $\vec{p}$ is the momentum. The generalized, matrix-valued mass term $M$ enters the Dirac equation in the form $\left(\mathrm{i} \gamma^{\mu} \partial_{\mu}-M\right) \psi(x)=0$. The $\gamma^{\mu}$ are $4 \times 4$ matrices that fulfill the relations $\left\{\gamma^{\mu}, \gamma^{\nu}\right\}=2 g^{\mu \nu}$, where we choose the space-time metric as $g^{\mu \nu}=\operatorname{diag}(1,-1,-1,-1)$. The $\partial_{\mu}$ denotes the partial derivative $\partial / \partial x^{\mu}$ with respect to the space-time coordinate $x^{\mu}=(t, \vec{x})$. It is quite surprising that a systematic presentation of the solutions of the generalized Dirac equations $\left(\mathrm{i} \gamma^{\mu} \partial_{\mu}-M\right) \psi(x)=0$, in the helicity basis [3], has not been recorded in the literature to the best of our knowledge. While the following discussion is somewhat technical, we believe that it will be beneficial to give their explicit form, in order to fix ideas for the following discussion.

We set $\hbar=c=\epsilon_{0}=1$ and use the Dirac matrices in the standard representation

$$
\begin{gathered}
\gamma^{0}=\beta=\left(\begin{array}{cc}
\mathbb{1}_{2 \times 2} & 0 \\
0 & -\mathbb{1}_{2 \times 2}
\end{array}\right), \quad \vec{\gamma}=\left(\begin{array}{cc}
0 & \vec{\sigma} \\
-\vec{\sigma} & 0
\end{array}\right), \\
\gamma^{5}=\left(\begin{array}{cc}
0 & \mathbb{1}_{2 \times 2} \\
\mathbb{1}_{2 \times 2} & 0
\end{array}\right),
\end{gathered}
$$


and define $\vec{\alpha}=\gamma^{0} \vec{\gamma}$. For the ordinary Dirac theory, one has $M=m_{1}$ (one should say more precisely $M=m_{1} \mathbb{1}_{4 \times 4}$ ) with a real mass $m_{1}$,

$$
\left(\mathrm{i} \gamma^{\mu} \partial_{\mu}-m_{1}\right) \psi(x)=0 .
$$

The dispersion relation is $E=\sqrt{\vec{p}^{2}+m_{1}^{2}}$. The corresponding Dirac Hamiltonian reads

$$
H^{(1)}=\vec{\alpha} \cdot \vec{p}+\beta m_{1}
$$

Extensions of the Dirac equation with pseudoscalar mass terms that contain the fifth current have been introduced in the literature. In [4], it is shown that for a mass term of the form $M=m_{1}+\mathrm{i} \gamma^{5} m_{2}$, the fermion propagator may obtain nontrivial gradient corrections already at the first order in derivative expansion, for a position-dependent mass. In that case, the fermion self-energy may contribute to a conceivable explanation for $\mathscr{C} \mathscr{P}$-violation during electroweak baryogenesis, as pointed out in [4]. We thus study the following generalized form of the tardyonic (subluminal) Dirac equation:

$$
\left(\mathrm{i} \gamma^{\mu} \partial_{\mu}-m_{1}-\mathrm{i} \gamma^{5} m_{2}\right) \psi(x)=0
$$

The dispersion relation is $E=\sqrt{\vec{p}^{2}+m_{1}^{2}+m_{2}^{2}}$. The Hermitian tardyonic Hamiltonian operator reads as

$$
H^{(t)}=\vec{\alpha} \cdot \vec{p}+\beta m_{1}+\mathrm{i} \beta \gamma^{5} m_{2}
$$

We may indicate a further motivation for our study; namely, the unitarity of the $S$ matrix implies the existence of useful relations [5] for the even powers $\left(m_{2}\right)^{2 n}$ obtained upon expanding a one-loop amplitude, formulated with a mass term $m_{1}+\mathrm{i} \gamma^{5} m_{2}$, in powers of $m_{2}$. This implies that a better understanding of the tardyonic equation with two mass terms could be of much more general interest.

It has not escaped our attention that the chiral transformation

$$
\mu \exp \left(\mathrm{i} \gamma^{5} \theta\right)=\mu \cos \theta+\mathrm{i} \gamma^{5} \mu \sin \theta=m_{1}+\mathrm{i} \gamma^{5} m_{2}
$$

connects the two Hamiltonians $H^{(1)}$ and $H^{(t)}$ for $m_{1}=\mu \cos \theta$ and $m_{2}=\mu \sin \theta$, but it is computationally easier and more instructive to consider the real and imaginary parts of the mass term separately.

Within a systematic approach to generalized Dirac equations with pseudoscalar mass terms, we also consider tachyonic (superluminal) mass terms of the form $M=\gamma^{5} m$ which induce a superluminal dispersion relation $E=\sqrt{\vec{p}^{2}-m^{2}}$. The corresponding generalized Dirac equation has been named the "tachyonic Dirac equation" and reads as follows [6-10]:

$$
\left(\mathrm{i} \gamma^{\mu} \partial_{\mu}-\gamma^{5} m\right) \psi(x)=0
$$

The corresponding Hamiltonian reads

$$
H_{5}=\vec{\alpha} \cdot \vec{p}+\beta \gamma^{5} m
$$

The relation $H_{5}=\gamma^{0} H_{5}^{+}(-\vec{r}) \gamma^{0}$ has been given in $[9,10]$. However, it is much more instructive to observe that $H_{5}$ is $\gamma^{5}$ Hermitian; that is, $H_{5}=\gamma^{5} H_{5}^{+} \gamma^{5}$. The concept of $\gamma^{5}$ Hermiticity is known in lattice theory $[11,12]$ and is otherwise called pseudo-Hermiticity [13-23].

An obvious generalization of the tachyonic case contains an imaginary mass and a $\gamma^{5}$ mass term,

$$
\left(\mathrm{i} \gamma^{\mu} \partial_{\mu}-\mathrm{i} m_{1}-\gamma^{5} m_{2}\right) \psi(x)=0 .
$$

The dispersion relation is $E=\sqrt{\vec{p}^{2}-m_{1}^{2}-m_{2}^{2}}$. The corresponding Hamiltonian reads

$$
H^{\prime}=\vec{\alpha} \cdot \vec{p}+\mathrm{i} \beta m_{1}+\beta \gamma^{5} m_{2}
$$

and is $\gamma^{5}$ Hermitian, $H^{\prime}=\gamma^{5} H^{\prime+} \gamma^{5}$. For $m_{2}=0$, (9) has been discussed in [24, 25].

It is our goal here to present the fundamental eigenspinors corresponding to the plane-wave solutions of (2), (4), (7), and (9) in a unified and systematic manner. Furthermore, we discuss the second-quantized versions of the fermionic theories described by the generalized Dirac equations. Anticipating part of the results, we may point out that the massless Dirac equation "interpolates" between the tardyonic equations (2) and (4) and the tachyonic equations (7) and (9). For zero mass, helicity and chirality are equal. Helicity and chirality "depart" from each other in very specific directions, when the tardyonic and tachyonic mass terms are "switched on," as we shall discuss in the following.

1.2. Tachyonic Dirac Equation and Neutrinos: Possible Connections. The tachyonic generalized Dirac equations (7) and (9) describe the motion of superluminal particles, which may either be important for astrophysical studies (neutrinos) or for artificially generated environments such as honeycomb photonic lattices in which pertinent dispersion relations become practically important [26]. The existence of superluminal particles would not falsify Einstein's theory of special relativity [27], which according to common wisdom is based on the following postulates. (i) The principle of relativity states that the laws of physics are the same for all observers in uniform motion relative to one another. (ii) The speed of light in a vacuum is the same for all observers, regardless of their relative motion or of the motion of the source of the light. Predictions of relativity theory regarding the relativity of simultaneity, time dilation, and length contraction would not change if superluminal particles did exist. Furthermore, as shown by Sudarshan et al. [28-31] and Feinberg [32, 33], the existence of tachyons, which are superluminal particles fulfilling a Lorentz-invariant dispersion relation $E^{2}=\vec{p}^{2}-$ $m_{v}^{2}$, is fully compatible with special relativity and Lorentz invariance. According to special relativity, it is forbidden to accelerate a particle "through" the light barrier (because $E=$ $m / \sqrt{1-v^{2}} \rightarrow \infty$ for $v \rightarrow 1$ ), but a genuinely superluminal particle remains superluminal upon Lorentz transformation. Significant problems are encountered when one attempts to quantize the tachyonic theories, but again, as shown in [9], these problems may not be as serious as previously thought. 
In particular, the so-called reinterpretation of solutions propagating into the past according to the Feynman prescription [31] is a cornerstone of modern field theory. Furthermore, it has been shown in [9] that tachyonic particles can be localized and equal-time anticommutators of the spin-1/2 tachyonic field involve an unfiltered Dirac- $\delta$ (see equation (37) of [9]).

Despite these arguments, we can say that, from the point of view of fundamental symmetries, accepting a superluminal neutrino would be equivalent to "an ugly duckling." Adding to the difficulties, we notice that recent experimental claims regarding the conceivable observation of highly superluminal neutrinos have turned out to be false. One may point out that a relative deviation $v=c(1+\delta)$ with $\delta \sim 10^{-5}$ at $E \sim 30 \mathrm{GeV}$, as claimed by some recent experimental collaborations, would correspond to a negative neutrino mass square in the order of $\sim-(100 \mathrm{MeV})^{2}$, if one assumes a Lorentzinvariant dispersion relation [34]. Still, there is at present no conclusive answer regarding the conceivable superluminality of at least one neutrino flavor [35-39], and it is intriguing that all available direct measurements of the neutrino mass square have resulted in negative expectation values, still compatible with zero within experimental uncertainty, whereas published experimental best estimates for the neutrino speed [40-43] have been superluminal, again still compatible with the speed of light within experimental error. The recent ICARUS result [43] is consistent with this trend [44-50]; the best estimate for the neutrino velocity is superluminal, but the deviation from $v_{v}=c$ is statistically insignificant. The OPERA collaboration [51] has indicated a preliminary, revised result of $(v-c) / c=\left(2.7 \pm 3.1(\text { stat })_{-2.8}^{+3.8}(\right.$ sys. $\left.)\right) \times 10^{-6}$. Neither subluminal nor superluminal propagation velocities are excluded based on the available experimental data. The "ugly duckling of a superluminal neutrino" is not beautiful; if we are to consider accepting it, then we should be able to hope that the emergence of at least one "added insight" should be the result of this operation.

Before we discuss the possible emergence of these benefits, let us include some historic remarks. According to reliable sources (Professor M. Fink from the University of Austin (Texas) was engaged in discussions with Professor J. A. Wheeler, who found the notion of a nonvanishing neutrino mass so unappealing that he discouraged experimentalists from undertaking any effort to measure the neutrino mass, based on arguments described in Section 1 (M. Fink, private communication, 2012.)), Professor J. A. Wheeler, in his later years at the University of Austin (Texas), used to argue that the neutrino has to be massless, necessarily, and that in his opinion, it could only be a massless Weyl particle with definite helicity (and chirality). Recently presented arguments [52] regarding the possibility of overtaking a subluminal, lefthanded neutrino, looking back and seeing a right-handed neutrino, were supposedly already used by Wheeler in order to dispel the conceivable existence of a neutrino mass term. If we assume that right-handed neutrinos are much more massive than their left-handed counterparts, then this leads to a paradox unless one assumes that all light, right-handed neutrinos are sterile. (The problem with a right-handed sterile massive neutrino is that for massive neutrinos, chirality and helicity are different; hence a $V-A$ coupling of the form $\gamma^{\mu}\left(1-\gamma^{5}\right)$ no longer vanishes for massive Dirac neutrinos if one uses the canonical eigenstates of the massive Dirac equation. One therefore has to invoke additional exotic mechanisms in order to ensure the "sterility" of the righthanded Dirac neutrinos.) Wheeler also disliked (Professor M. Fink from the University of Austin (Texas) was engaged in discussions with Professor J. A. Wheeler, who found the notion of a nonvanishing neutrino mass so unappealing that he discouraged experimentalists from undertaking any effort to measure the neutrino mass, based on arguments described in Section 1 (M. Fink, private communication, 2012.)) the notion of a Majorana neutrino, arguing that the charge conjugation invariance condition imposed on the Majorana particle precludes the existence of plane-wave solutions to the Majorana equation and maximally violates lepton number. Again, these arguments (We are grateful to Professor M. Fink for the clarification and confirmation.) are in full agreement with those recently given in [52].

The original standard model thus called for manifestly massless neutrinos. The commonly accepted observation of neutrino oscillations precludes the possibility that all three generations of neutrino mass eigenstates are massless. Lepton number conservation is based on the global gauge symmetry $\psi \rightarrow \psi \exp (\mathrm{i} \Lambda$ ), applied simultaneously to all lepton fields. A Majorana neutrino would destroy lepton number as a global symmetry but solve the "autobahn paradox," because a Majorana neutrino would be equal to its own antiparticle and thus, looking back, the right-handed neutrino state would consist of the same particle = antiparticle.

On the other hand, if we assume that the neutrino is described by the tachyonic Dirac equation, then the following statements are valid.

(i) Statement 1: we can properly assign lepton number and use plane-wave eigenstates for incoming and outgoing particles, while allowing for nonvanishing mass terms and thus mass square differences among the neutrino mass (not flavor) eigenstates.

(ii) Statement 2: there is a natural resolution for the "autobahn paradox" because a left-handed spacelike neutrino always remains spacelike upon Lorentz transformation and cannot be overtaken.

(iii) Statement 3: the right-handed particle and lefthanded antiparticle states are suppressed due to negative Fock-space norm.

(iv) Statement 4: at least qualitatively, tachyonic neutrinos could yield an explanation for a repulsive force on intergalactic distance scales as they are repulsed, like all tachyons, by gravitational interactions (dark energy).

Pauli [53] postulated the existence of neutrinos, on the basis of the conservation of angular momentum and energy, and also introduced pseudo-Hermitian operators [13]. Here, we describe conceivable connections of neutrino physics and pseudo-Hermitian operators. Final clarification can only come from experiment. When in 1956, Reines and Cowan [54] discovered the electron neutrino, two and a half years 
before Pauli's death, Pauli replied [55] by telegram "Thanks for message. Everything comes to him who knows how to wait. Pauli." In defense of the tachyonic hypothesis, we would like to stress that a tachyonic Dirac neutrino would allow us to retain lepton number conservation as a symmetry of nature. We would thus like to write up these thoughts in the current paper, with attention to detail. We should point out that our approach fully conserves Lorentz invariance, in contrast to the extensions of the Standard Model based on Lorentzviolating terms which can otherwise lead to superluminal propagation (see Tables 11 and 13 of [56]).

Units with $\hbar=c=\epsilon_{0}=1$ are used throughout the paper. The organization is as follows: in Section 2, we discuss massless and tardyonic theories. Tachyonic extensions of the Dirac equation are discussed in Section 3. Connections of the fundamental tensor sums over the eigenspinors with the derivation of the time-ordered propagator are analyzed in Section 4. A candidate for dark energy is presented in Section 5. Conclusions are reserved for Section 6.

\section{Generalized Dirac Equations: Massless and Tardyonic Theories}

2.1. Massless Dirac Theory. The massless Dirac equation and the massless Dirac Hamiltonian read as

$$
\mathrm{i} \gamma^{\mu} \partial_{\mu} \psi(x)=0, \quad H_{0}=\vec{\alpha} \cdot \vec{p} .
$$

We note that $H_{0}$ is both Hermitian as well as $\gamma^{5}$ Hermitian; that is, $H_{0}=\gamma^{5} H_{0}^{+} \gamma^{5}$. The dispersion relation is $E=|\vec{k}|$. With $k^{\mu}=(E, \vec{k})$, we seek positive-energy and negative-energy solutions of the form

$$
\psi(x)=u_{\sigma}(\vec{k}) \exp (-\mathrm{i} k \cdot x), \quad \phi(x)=v_{\sigma}(\vec{k}) \exp (\mathrm{i} k \cdot x),
$$

where $\sigma= \pm$ denotes a quantum number which is equal to the helicity for positive-energy states and equal to the negative of the helicity for negative-energy states. With $k=\gamma^{\mu} k_{\mu}$, we have $k u_{ \pm}(k)=k v_{ \pm}(k)=0$. In the massless limit, the solutions to the Dirac equation are given as (see Chapter 2 of [57])

$$
\begin{gathered}
u_{+}(\vec{k})=\frac{1}{\sqrt{2}}\left(\begin{array}{c}
a_{+}(\vec{k}) \\
a_{+}(\vec{k})
\end{array}\right), \quad u_{-}(\vec{k})=\frac{1}{\sqrt{2}}\left(\begin{array}{c}
a_{-}(\vec{k}) \\
-a_{-}(\vec{k})
\end{array}\right), \\
v_{+}(\vec{k})=-u_{+}(\vec{k}), \quad v_{-}(\vec{k})=-u_{-}(\vec{k}) .
\end{gathered}
$$

The well-known helicity spinors are recalled as

$$
\begin{gathered}
a_{+}(\vec{k})=\left(\begin{array}{c}
\cos \left(\frac{\theta}{2}\right) \\
\sin \left(\frac{\theta}{2}\right) \mathrm{e}^{\mathrm{i} \varphi}
\end{array}\right), \\
a_{-}(\vec{k})=\left(\begin{array}{c}
-\sin \left(\frac{\theta}{2}\right) \mathrm{e}^{-\mathrm{i} \varphi} \\
\cos \left(\frac{\theta}{2}\right)
\end{array}\right) .
\end{gathered}
$$

These fulfill the fundamental relations $(\vec{\sigma} \cdot \widehat{\vec{k}}) a_{\sigma}(\vec{k})=\sigma a_{\sigma}(\vec{k})$, as well as $\sum_{\sigma} a_{\sigma}(\vec{k}) \otimes a_{\sigma}^{+}(\vec{k})=\mathbb{1}_{2}$ and $\sum_{\sigma} \sigma a_{\sigma}(\vec{k}) \otimes a_{\sigma}^{+}(\vec{k})=\vec{\sigma} \cdot \widehat{\vec{k}}$, where $\widehat{\vec{k}}=\vec{k} /|\vec{k}|$ and the sum over $\sigma$ is over the values \pm 1 . The sums over the fundamental bispinors $u$ and $v$ fulfill the following sum rules:

$$
\text { Sum rule I: } \sum_{\sigma} 2|\vec{k}| u_{\sigma}(\vec{k}) \otimes \bar{u}_{\sigma}(\vec{k})=\not k,
$$

as well as

$$
\text { Sum rule II: } \sum_{\sigma} 2|\vec{k}|(-\sigma) u_{\sigma}(\vec{k}) \otimes \bar{u}_{\sigma}(\vec{k}) \gamma^{5}=\not k,
$$

Sum rule I can be obtained by a quick explicit calculation, and sum rule II holds because in the massless limit, helicity equals \pm chirality (positive sign for positive energy, negative sign for negative energy). We denote the Dirac adjoint by $\bar{u}_{\sigma}(\vec{k})=$ $u_{\sigma}^{+}(\vec{k}) \gamma^{0}$. One can easily check by an explicit calculation that $\bar{u}_{\sigma}(\vec{k}) \gamma^{5}=\left(\gamma^{5} \gamma^{0} u_{\sigma}(\vec{k})\right)^{+}=(-\sigma) \bar{u}_{\sigma}(\vec{k})$ and $\bar{v}_{\sigma}(\vec{k}) \gamma^{5}=$ $\left(\gamma^{5} \gamma^{0} v_{\sigma}(\vec{k})\right)^{+}=(-\sigma) \bar{v}_{\sigma}(\vec{k})$. We can thus introduce a factor $(-\sigma)^{2}=1$ under the summation over spins in (15) and replace one of the factors $(-\sigma)$ by a multiplication of the Dirac adjoint spinor from the right by the fifth current. The Lorentzinvariant normalization of the massless solutions vanishes; that is, $\bar{u}_{\sigma}(\vec{k}) u_{\sigma}(\vec{k})=\bar{v}_{\sigma}(\vec{k}) v_{\sigma}(\vec{k})=0$.

Eigenstates of the massless Hamiltonian $H_{0}=\vec{\alpha} \cdot \vec{p}$ have to be eigenstates of the chirality operator $\gamma^{5}$ because the chirality commutes with the Hamiltonian, in the sense that $\left[\gamma^{5}, H_{0}\right]=$ 0 . Furthermore,

$$
H_{0}=\vec{\alpha} \cdot \vec{p}=|\vec{p}| \gamma^{5}\left(\frac{\vec{\Sigma} \cdot \vec{p}}{|\vec{p}|}\right),
$$

where $\vec{\Sigma}=\gamma^{5} \vec{\alpha}$ is the vector of $4 \times 4$ spin matrices, and the helicity operator is identified as $\vec{\Sigma} \cdot \vec{p} /|\vec{p}|$. Let $\lambda_{1}$ be the eigenvalue of chirality and let $\lambda_{2}$ be the eigenvalue of the helicity operator. Then, the eigenvalue of the Hamiltonian is $E_{0}=|\vec{p}| \lambda_{1} \lambda_{2}$. Since $\lambda_{1}= \pm 1$ and $\lambda_{2}= \pm 1$, we easily recover the known fact that helicity equals chirality for positive energy, whereas the relation is reversed for negativeenergy states (see also Chapter 2.4 of [57]). We are aware of the fact that the considerations reported in the current section partly refer to the literature, but we give them in some detail because they are essential for the following considerations.

2.2. Massive Dirac Theory. We start from the ordinary Dirac equation given in (2), which reads $\left(\mathrm{i} \gamma^{\mu} \partial_{\mu}-m_{1}\right) \psi(x)=0$. In the helicity basis, the fundamental spinor solutions read as

$$
\begin{gathered}
\psi(x)=U_{ \pm}^{(1)}(\vec{k}) \exp (-\mathrm{i} k \cdot x), \\
\phi(x)=V_{ \pm}^{(1)}(\vec{k}) \exp (\mathrm{i} k \cdot x) .
\end{gathered}
$$

The algebraic relations that have to be fulfilled by the bispinor amplitudes $U_{ \pm}^{(1)}(\vec{k})$ and $V_{ \pm}^{(1)}(\vec{k})$ read as follows:

$$
\left(k-m_{1}\right) U_{ \pm}^{(1)}(\vec{k})=0, \quad\left(k+m_{1}\right) V_{ \pm}^{(1)}(\vec{k})=0 .
$$


The dispersion relation is $E^{(1)}=\sqrt{\vec{k}^{2}+m_{1}^{2}}$. In the helicity basis, the solutions of (19) with a tardyonic $m_{1}$ mass term are easily written down, using the identity $\left(k-m_{1}\right)\left(k+m_{1}\right)=k^{2}-$ $m_{1}^{2}=0$. With an appropriate normalization factor and after some algebraic simplification, the positive-energy solutions read as follows:

$$
\begin{aligned}
& U_{+}^{(1)}(\vec{k})=\frac{\left(k+m_{1}\right) u_{+}(\vec{k})}{\sqrt{\left(E^{(1)}-|\vec{k}|\right)^{2}+m_{1}^{2}}} \\
& =\left(\begin{array}{c}
\sqrt{\frac{E^{(1)}+m_{1}}{2 E^{(1)}}} a_{+}(\vec{k}) \\
\sqrt{\frac{E^{(1)}-m_{1}}{2 E^{(1)}}} a_{+}(\vec{k})
\end{array}\right), \\
& U_{-}^{(1)}(\vec{k})=\frac{\left(k+m_{1}\right) u_{-}(\vec{k})}{\sqrt{\left(E^{(1)}-|\vec{k}|\right)^{2}+m_{1}^{2}}} \\
& =\left(\begin{array}{c}
\sqrt{\frac{E^{(1)}+m_{1}}{2 E^{(1)}}} a_{-}(\vec{k}) \\
-\sqrt{\frac{E^{(1)}-m_{1}}{2 E^{(1)}} a_{-}}(\vec{k})
\end{array}\right) .
\end{aligned}
$$

The negative-energy eigenstates of the tardyonic equations in the helicity basis are given as

$$
\begin{aligned}
V_{+}^{(1)}(\vec{k}) & =\frac{\left(m_{1}-\not k\right) v_{+}(\vec{k})}{\sqrt{\left(E^{(1)}-|\vec{k}|\right)^{2}+m_{1}^{2}}} \\
& =\left(\begin{array}{l}
-\sqrt{\frac{E^{(1)}-m_{1}}{2 E^{(1)}}} a_{+}(\vec{k}) \\
-\sqrt{\frac{E^{(1)}+m_{1}}{2 E^{(1)}} a_{+}}(\vec{k})
\end{array}\right), \\
V_{-}^{(1)}(\vec{k}) & =\frac{\left(m_{1}-\not k\right) v_{-}(\vec{k})}{\sqrt{\left(E^{(1)}-|\vec{k}|\right)^{2}+m_{1}^{2}}} \\
& =\left(\begin{array}{l}
-\sqrt{\frac{E^{(1)}-m_{1}}{2 E^{(1)}}} a_{-}(\vec{k}) \\
\sqrt{\frac{E^{(1)}+m_{1}}{2 E^{(1)}} a_{-}}(\vec{k})
\end{array}\right) .
\end{aligned}
$$

These solutions are consistent with those given in [3] and in Chapter 23 of [58], and the normalizations are $(\sigma= \pm)$

$$
U_{\sigma}^{(1)+}(\vec{k}) U_{\sigma}^{(1)}(\vec{k})=V_{\sigma}^{(1)+}(\vec{k}) V_{\sigma}^{(1)}(\vec{k})=1 .
$$

One can change the normalization according to

$$
\begin{aligned}
& \mathcal{U}_{\sigma}^{(1)}(\vec{k})=\left(\frac{E^{(1)}}{m_{1}}\right)^{1 / 2} U_{\sigma}^{(1)}(\vec{k}), \\
& \mathscr{V}_{\sigma}^{(1)}(\vec{k})=\left(\frac{E^{(1)}}{m_{1}}\right)^{1 / 2} V_{\sigma}^{(1)}(\vec{k}) .
\end{aligned}
$$

The Lorentz-invariant normalization is equal to one for the fundamental positive-energy bispinors and equal to minus one for the fundamental negative-energy bispinors,

$$
\begin{gathered}
\overline{\mathcal{U}}_{\sigma}^{(1)}(\vec{k}) \mathcal{U}_{\sigma}^{(1)}(\vec{k})=1, \\
\overline{\mathscr{V}}_{\sigma}^{(1)}(\vec{k}) \mathscr{V}_{\sigma}^{(1)}(\vec{k})=-1 .
\end{gathered}
$$

A little algebra is sufficient to reproduce the following known sums over bispinors:

$$
\begin{aligned}
& \sum_{\sigma} \mathcal{U}_{\sigma}^{(1)}(\vec{k}) \otimes \overline{\mathcal{U}}_{\sigma}^{(1)}(\vec{k})=\frac{k+m_{1}}{2 m_{1}}, \\
& \sum_{\sigma} \mathscr{V}_{\sigma}^{(1)}(\vec{k}) \otimes \overline{\mathscr{V}}_{\sigma}^{(1)}(\vec{k})=\frac{k-m_{1}}{2 m_{1}} .
\end{aligned}
$$

In accordance with general wisdom of the tardyonic case, these do not involve a helicity-dependent prefactor. The sum rule (25) is of type I (see (15)).

2.3. Two Tardyonic Mass Terms. Inspired by the discussion in Section 1, we consider an equation with two tardyonic mass terms, which has already been indicated in (2) and reads $\left(\mathrm{i} \gamma^{\mu} \partial_{\mu}-m_{1}-\mathrm{i} \gamma^{5} m_{2}\right) \psi^{(t)}(x)=0$. For the corresponding bispinors in the fundamental plane-wave solutions, this implies that

$$
\begin{gathered}
\left(k-m_{1}-\mathrm{i} \gamma^{5} m_{2}\right) U_{ \pm}^{(t)}(\vec{k})=0, \\
\left(-k-m_{1}-\mathrm{i} \gamma^{5} m_{2}\right) V_{ \pm}^{(t)}(\vec{k})=0 .
\end{gathered}
$$

The dispersion relation is $E^{(t)}=\sqrt{\vec{k}^{2}+m_{1}^{2}+m_{2}^{2}}$. The fundamental positive-energy bispinors read as follows:

$$
\begin{aligned}
U_{+}^{(t)}(\vec{k}) & =\frac{\left(k+m_{1}-\mathrm{i} \gamma^{5} m_{2}\right) u_{+}(\vec{k})}{\sqrt{\left(E^{(t)}-|\vec{k}|\right)^{2}+m_{1}^{2}+m_{2}^{2}}} \\
& =\left(\begin{array}{c}
\frac{m_{1}-\mathrm{i} m_{2}+E^{(t)}-|\vec{k}|}{\sqrt{\left(E^{(t)}-|\vec{k}|\right)^{2}+m_{1}^{2}+m_{2}^{2}}} \frac{a_{+}(\vec{k})}{\sqrt{2}} \\
\frac{m_{1}-\mathrm{i} m_{2}-E^{(t)}+|\vec{k}|}{\sqrt{\left(E^{(t)}-|\vec{k}|\right)^{2}+m_{1}^{2}+m_{2}^{2}}} \frac{a_{+}}{\sqrt{2}}
\end{array}\right),
\end{aligned}
$$




$$
\begin{aligned}
U_{-}^{(t)}(\vec{k}) & =\frac{\left(k+m_{1}-\mathrm{i} \gamma^{5} m_{2}\right) u_{-}(\vec{k})}{\sqrt{\left(E^{(t)}-|\vec{k}|\right)^{2}+m_{1}^{2}+m_{2}^{2}}} \\
& =\left(\begin{array}{c}
\frac{m_{1}+\mathrm{i} m_{2}+E^{(t)}-|\vec{k}|}{\sqrt{\left(E^{(t)}-|\vec{k}|\right)^{2}+m_{1}^{2}+m_{2}^{2}}} \frac{a_{-}(\vec{k})}{\sqrt{2}} \\
\frac{-m_{1}-\mathrm{i} m_{2}+E^{(t)}-|\vec{k}|}{\sqrt{\left(E^{(t)}-|\vec{k}|\right)^{2}+m_{1}^{2}+m_{2}^{2}}} \frac{a_{-}}{\sqrt{2}}
\end{array}\right) .
\end{aligned}
$$

The negative-energy eigenstates of the equation with two tardyonic mass terms are given as

$$
\begin{aligned}
V_{+}^{(t)}(\vec{k}) & =\frac{\left(-k-\mathrm{i} \gamma^{5} m_{2}+m_{1}\right) v_{+}(\vec{k})}{\sqrt{\left(E^{(t)}-|\vec{k}|\right)^{2}+m_{1}^{2}+m_{2}^{2}}} \\
& =\left(\begin{array}{c}
\frac{-m_{1}+\mathrm{i} m_{2}+E^{(t)}-|\vec{k}|}{\sqrt{\left(E^{(t)}-|\vec{k}|\right)^{2}+m_{1}^{2}+m_{2}^{2}}} \frac{a_{+}(\vec{k})}{\sqrt{2}} \\
\frac{-m_{1}+\mathrm{i} m_{2}-E^{(t)}+|\vec{k}|}{\sqrt{\left(E^{(t)}-|\vec{k}|\right)^{2}+m_{1}^{2}+m_{2}^{2}}} \frac{a_{+}}{\sqrt{k}}
\end{array}\right),
\end{aligned}
$$

for negative helicity (positive chirality in the massless limit) and

$$
\begin{aligned}
V_{-}^{(t)}(\vec{k}) & =\frac{\left(-\not k-\mathrm{i} \gamma^{5} m_{2}+m_{1}\right) v_{-}(\vec{k})}{\sqrt{\left(E^{(t)}-|\vec{k}|\right)^{2}+m_{1}^{2}+m_{2}^{2}}} \\
& =\left(\begin{array}{c}
\frac{-m_{1}-\mathrm{i} m_{2}+E^{(t)}-|\vec{k}|}{\sqrt{\left(E^{(t)}-|\vec{k}|\right)^{2}+m_{1}^{2}+m_{2}^{2}}} \frac{a_{-}(\vec{k})}{\sqrt{2}} \\
\frac{m_{1}+\mathrm{i} m_{2}+E^{(t)}-|\vec{k}|}{\sqrt{\left(E^{(t)}-|\vec{k}|\right)^{2}+m_{1}^{2}+m_{2}^{2}}} \frac{a_{-}(\vec{k})}{\sqrt{2}}
\end{array}\right),
\end{aligned}
$$

for positive helicity (negative chirality in the massless limit). In the massless limit (first $E^{(t)} \rightarrow|\vec{k}|$, then $m_{2} \rightarrow 0$, and then $m_{1} \rightarrow 0$ ), we again reproduce the massless solutions $U_{+}^{(t)}(\vec{k}) \rightarrow u_{+}(\vec{k}), U_{-}^{(t)}(\vec{k}) \rightarrow u_{-}(\vec{k}), V_{+}^{(t)}(\vec{k}) \rightarrow v_{+}(\vec{k})$ and $V_{-}^{(t)}(\vec{k}) \rightarrow v_{-}(\vec{k})$. Of course, in the limit $m_{1} \rightarrow 0$, one also has to expand the normalization denominators in powers of $m_{1}$. For $m_{2}=0$ the solutions (27a), (27b), (28a), and (28b) reduce to the solutions of the ordinary Dirac equation in (20a), (20b), (21a), and (21b) and can be expanded in $m_{2}$ to yield corrections to the ordinary Dirac equation for small $m_{2}$; that is, $m_{1} \gg m_{2}$. The states are normalized with respect to the condition

$$
U_{\sigma}^{(t)+}(\vec{k}) U_{\sigma}^{(t)}(\vec{k})=V_{\sigma}^{(t)+}(\vec{k}) V_{\sigma}^{(t)}(\vec{k})=1
$$

In the normalization

$$
\begin{aligned}
& \mathcal{U}_{\sigma}^{(t)}(\vec{k})=\left(\frac{E^{(t)}}{m_{1}}\right)^{1 / 2} U_{\sigma}^{(t)}(\vec{k}), \\
& \mathscr{V}_{\sigma}^{(t)}(\vec{k})=\left(\frac{E^{(t)}}{m_{1}}\right)^{1 / 2} V_{\sigma}^{(t)}(\vec{k}),
\end{aligned}
$$

the positive-energy solutions acquire a "positive Lorentzinvariant norm," whereas the negative-energy solutions have "negative Lorentz-invariant norm,"

$$
\overline{\mathcal{U}}_{\sigma}^{(t)}(\vec{k}) \mathcal{U}_{\sigma}^{(t)}(\vec{k})=1, \quad \overline{\mathscr{V}}_{\sigma}^{(t)}(\vec{k}) \mathscr{V}_{\sigma}^{(t)}(\vec{k})=-1
$$

After some algebra, one can derive the following sums over bispinors:

$$
\begin{aligned}
& \sum_{\sigma} U_{\sigma}^{(t)}(\vec{k}) \otimes \bar{U}_{\sigma}^{(t)}(\vec{k})=\frac{k+m_{1}-\mathrm{i} \gamma^{5} m_{2}}{2 m_{1}}, \\
& \sum_{\sigma} V_{\sigma}^{(t)}(\vec{k}) \otimes \bar{V}_{\sigma}^{(t)}(\vec{k})=\frac{k-m_{1}+\mathrm{i} \gamma^{5} m_{2}}{2 m_{1}} .
\end{aligned}
$$

These are easily identified as the positive- and negativeenergy projectors. The sum rules do not involve helicitydependent prefactor and are of type I (see (15)).

The solutions (27a), (27b), (28a), and (28b) approach the massless solutions if one replaces $m_{2} \rightarrow 0$ first and then let $m_{1} \rightarrow 0$. They are thus useful for systems where the $m_{1}$ mass is greater than $m_{2}$. For $m_{2} \gg m_{1}$, one would like to calculate solutions that approach the massless case for the sequence $m_{1} \rightarrow 0$, then $m_{2} \rightarrow 0$. These read as follows:

$$
U_{\sigma}^{\prime(t)}(\vec{k})=\mathrm{i} \sigma U_{\sigma}^{(t)}(\vec{k}), \quad V_{\sigma}^{\prime(t)}(\vec{k})=\mathrm{i} \sigma V_{\sigma}^{(t)}(\vec{k})
$$

In comparison to the solutions (27a), (27b), (28a), and (28b), they acquire a nontrivial phase factor.

\section{Generalized Dirac Equations: Tachyonic Mass Terms}

3.1. Tachyonic Dirac Equation. The tachyonic Dirac equation is given in (7) and reads $\left(\mathrm{i} \gamma^{\mu} \partial_{\mu}-\gamma^{5} m\right) \psi(x)=0$. The fundamental bispinors entering the equations fulfill the equations

$$
\left(k-\gamma^{5} m\right) U_{ \pm}(\vec{k})=0, \quad\left(k+\gamma^{5} m\right) V_{ \pm}(\vec{k})=0 .
$$


Using $\left(k-\gamma^{5} m\right)\left(k-\gamma^{5} m\right)=k^{2}+m^{2}$ and some algebra, the prefactors in the fundamental bispinors (for positive energy) take a very simple form,

$$
\begin{gathered}
U_{+}(\vec{k})=\frac{\left(\gamma^{5} m-\not k\right) u_{+}(\vec{k})}{\sqrt{(E-|\vec{k}|)^{2}+m^{2}}}=\left(\begin{array}{c}
\sqrt{\frac{|\vec{k}|+m}{2|\vec{k}|}} a_{+}(\vec{k}) \\
\sqrt{\frac{|\vec{k}|-m}{2|\vec{k}|}} a_{+}(\vec{k})
\end{array}\right), \\
U_{-}(\vec{k})=\frac{\left(k-\gamma^{5} m\right) u_{-}(\vec{k})}{\sqrt{(E-|\vec{k}|)^{2}+m^{2}}}=\left(\begin{array}{c}
\sqrt{\frac{|\vec{k}|-m}{2|\vec{k}|}} a_{-}(\vec{k}) \\
-\sqrt{\frac{|\vec{k}|+m}{2|\vec{k}|}} a_{-}(\vec{k})
\end{array}\right) .
\end{gathered}
$$

For negative energy, the solutions read as follows:

$$
\begin{gathered}
V_{+}(\vec{k})=\frac{\left(\gamma^{5} m+\not k\right) v_{+}(\vec{k})}{\sqrt{(E-|\vec{k}|)^{2}+m^{2}}}=\left(\begin{array}{l}
-\sqrt{\frac{|\vec{k}|-m}{2|\vec{k}|}} a_{+}(\vec{k}) \\
-\sqrt{\frac{|\vec{k}|+m}{2|\vec{k}|}} a_{+}(\vec{k})
\end{array}\right), \\
V_{-}(\vec{k})=\frac{\left(-k-\gamma^{5} m\right) v_{-}(\vec{k})}{\sqrt{(E-|\vec{k}|)^{2}+m^{2}}}=\left(\begin{array}{l}
-\sqrt{\frac{|\vec{k}|+m}{2|\vec{k}|}} a_{+}(\vec{k}) \\
\sqrt{\frac{|\vec{k}|-m}{2|\vec{k}|}} a_{+}(\vec{k})
\end{array}\right) .
\end{gathered}
$$

The normalization condition is $(\sigma= \pm)$

$$
U_{\sigma}^{+}(\vec{k}) U_{\sigma}(\vec{k})=V_{\sigma}^{+}(\vec{k}) V_{\sigma}(\vec{k})=1 .
$$

One can change to Lorentz-invariant normalization by a multiplication with $(|\vec{k}| / m)^{1 / 2}$,

$$
\begin{aligned}
& \mathscr{U}_{\sigma}(\vec{k})=\left(\frac{|\vec{k}|}{m}\right)^{1 / 2} U_{\sigma}(\vec{k}), \\
& \mathscr{V}_{\sigma}(\vec{k})=\left(\frac{|\vec{k}|}{m}\right)^{1 / 2} V_{\sigma}(\vec{k}) .
\end{aligned}
$$

The "calligraphic" spinors fulfill the following helicity-dependent normalizations:

$$
\bar{U}_{\sigma}(\vec{k}) \mathscr{U}_{\sigma}(\vec{k})=\sigma, \quad \overline{\mathscr{V}}_{\sigma}(\vec{k}) \mathscr{V}_{\sigma}(\vec{k})=-\sigma,
$$

where we observe that $\sigma$ is a good quantum number because the helicity operator commutes with the Hamiltonian (8).
The sum rule fulfilled by the fundamental plane-wave spinors is of type II (see (16)). For the positive-energy spinors, we have

$$
\sum_{\sigma}(-\sigma) \mathcal{U}_{\sigma}(\vec{k}) \otimes \overline{\mathcal{U}}_{\sigma}(\vec{k}) \gamma^{5}=\frac{k-\gamma^{5} m}{2 m}
$$

where for the negative-energy spinors, the sum rule reads

$$
\sum_{\sigma}(-\sigma) \mathscr{V}_{\sigma}(\vec{k}) \otimes \overline{\mathscr{V}}_{\sigma}(\vec{k}) \gamma^{5}=\frac{k+\gamma^{5} m}{2 m}
$$

The expressions on the right-hand sides are the positive- and negative-energy projectors.

3.2. Two Tachyonic Mass Terms. We study the equation $\left(\mathrm{i} \gamma^{\mu} \partial_{\mu}-\mathrm{i} m_{1}-\gamma^{5} m_{2}\right) \psi(x)=0$, as given in (9). The fundamental spinors, which we denote $U_{ \pm}^{\prime}(\vec{k})$ and $V_{ \pm}^{\prime}(\vec{k})$, fulfill the following equations:

$$
\begin{aligned}
& \left(k-\mathrm{i} m_{1}-\gamma^{5} m_{2}\right) U_{ \pm}^{\prime}(\vec{k})=0, \\
& \left(k+\mathrm{i} m_{1}+\gamma^{5} m_{2}\right) V_{ \pm}^{\prime}(\vec{k})=0 .
\end{aligned}
$$

The positive-energy solutions are obtained using the identity $\left(k-\mathrm{i} m_{1}-\gamma^{5} m_{2}\right)\left(k+\mathrm{i} m_{1}-\gamma^{5} m_{2}\right)=k^{2}+m_{1}^{2}+m_{2}^{2}$. With $E^{\prime}=\sqrt{\vec{k}^{2}-m_{1}^{2}-m_{2}^{2}}$, they read as follows:

$$
\begin{gathered}
U_{+}^{\prime}(\vec{k})=\left(\begin{array}{c}
\frac{\mathrm{i} m_{1}+m_{2}-E^{\prime}+|\vec{k}|}{\sqrt{\left(E^{\prime}-|\vec{k}|\right)^{2}+m_{1}^{2}+m_{2}^{2}}} \frac{a_{+}(\vec{k})}{\sqrt{2}} \\
\frac{\mathrm{i} m_{1}+m_{2}+E^{\prime}-|\vec{k}|}{\sqrt{\left(E^{\prime}-|\vec{k}|\right)^{2}+m_{1}^{2}+m_{2}^{2}}} \frac{a_{+}(\vec{k})}{\sqrt{2}}
\end{array}\right), \\
U_{-}^{\prime}(\vec{k})=\left(\begin{array}{l}
\frac{\mathrm{i} m_{1}+m_{2}+E^{\prime}-|\vec{k}|}{\sqrt{\left(E^{\prime}-|\vec{k}|\right)^{2}+m_{1}^{2}+m_{2}^{2}}} \frac{a_{-}(\vec{k})}{\sqrt{2}} \\
\frac{-\mathrm{i} m_{1}-m_{2}+E^{\prime}-|\vec{k}|}{\sqrt{\left(E^{\prime}-|\vec{k}|\right)^{2}+m_{1}^{2}+m_{2}^{2}}} \frac{a_{-}(\vec{k})}{\sqrt{2}}
\end{array}\right) .
\end{gathered}
$$


The negative-energy solutions for the tachyonic equation with two mass terms are given as

$$
\begin{aligned}
& V_{+}^{\prime}(\vec{k})=\left(\begin{array}{c}
\frac{\mathrm{i} m_{1}-m_{2}-E^{\prime}+|\vec{k}|}{\sqrt{\left(E^{\prime}-|\vec{k}|\right)^{2}+m_{1}^{2}+m_{2}^{2}}} \frac{a_{+}(\vec{k})}{\sqrt{2}} \\
\frac{\mathrm{i} m_{1}-m_{2}-E^{\prime}-|\vec{k}|}{\sqrt{\left(E^{\prime}-|\vec{k}|\right)^{2}+m_{1}^{2}+m_{2}^{2}}} \frac{a_{+}(\vec{k})}{\sqrt{2}}
\end{array}\right), \\
& V_{-}^{\prime}(\vec{k})=\left(\begin{array}{c}
\frac{-\mathrm{i} m_{1}-m_{2}+E^{\prime}-|\vec{k}|}{\sqrt{\left(E^{\prime}-|\vec{k}|\right)^{2}+m_{1}^{2}+m_{2}^{2}}} \frac{a_{-}(\vec{k})}{\sqrt{2}} \\
\frac{\mathrm{i} m_{1}+m_{2}+E^{\prime}-|\vec{k}|}{\sqrt{\left(E^{\prime}-|\vec{k}|\right)^{2}+m_{1}^{2}+m_{2}^{2}}} \frac{a_{-}(\vec{k})}{\sqrt{2}}
\end{array}\right) .
\end{aligned}
$$

The normalization condition is $U_{\sigma}^{\prime+}(\vec{k}) U_{\sigma}^{\prime}(\vec{k})=V_{\sigma}^{\prime+}(\vec{k}) V_{\sigma}^{\prime}(\vec{k})=$ 1 . We use a definition of the "calligraphic" spinors analogous to $(38)$,

$$
\begin{aligned}
& \mathcal{U}_{\sigma}^{\prime}(\vec{k})=\left(\frac{|\vec{k}|}{m_{2}}\right)^{1 / 2} U_{\sigma}^{\prime}(\vec{k}), \\
& \mathscr{V}_{\sigma}^{\prime}(\vec{k})=\left(\frac{|\vec{k}|}{m_{2}}\right)^{1 / 2} V_{\sigma}^{\prime}(\vec{k}) .
\end{aligned}
$$

In analogy to (40a) and (40b), a sum rule of type II (see (16)) is fulfilled by the fundamental plane-wave spinors,

$$
\begin{aligned}
& \sum_{\sigma}(-\sigma) \mathcal{U}_{\sigma}^{\prime}(\vec{k}) \otimes \overrightarrow{\mathcal{U}}_{\sigma}^{\prime}(\vec{k}) \gamma^{5}=\frac{k+\mathrm{i} m_{1}-\gamma^{5} m_{2}}{2 m_{2}}, \\
& \sum_{\sigma}(-\sigma) \mathscr{V}_{\sigma}^{\prime}(\vec{k}) \otimes \overrightarrow{\mathscr{V}}_{\sigma}^{\prime}(\vec{k}) \gamma^{5}=\frac{k-\mathrm{i} m_{1}+\gamma^{5} m_{2}}{2 m_{2}} .
\end{aligned}
$$

We thus obtain that the desired projectors onto positive- and negative-energy solutions for the Dirac equation with two tachyonic mass terms (9). The generalized equation $\left(\mathrm{i} \gamma^{\mu} \partial_{\mu}-\right.$ $\left.\mathrm{i} m_{1}-\gamma^{5} m_{2}\right) \psi(x)=0$ is fully compatible with the Cliffordalgebra-based approach recently described in [59].

\section{Theorems for Generalized Dirac Fields}

4.1. Spinor Sums and Time-Ordered Propagator. Our central postulate regarding the quantized fermionic theory is that the time-ordered vacuum expectation value of the field operators should yield the time-ordered (Feynman) propagator, which, in the momentum representation, is equal to the inverse of the Hamiltonian (upon multiplication with $\gamma^{0}$ ). This postulate implies that under rather general assumptions regarding the mathematical form of the elementary field anticommutators, sum rules have to be fulfilled by the tensor sums over the fundamental spinor solutions. It is perhaps not surprising that these sum rules are precisely of the form investigated in Sections 2 and 3 of this paper.
For definiteness, we consider the solution of the tachyonic Dirac equation (Section 3.1). The generalization to other generalized Dirac equations is straightforward. We start from the field operator [9]

$$
\begin{aligned}
\psi(x)=\int & \frac{\mathrm{d}^{3} k}{(2 \pi)^{3}} \frac{m}{E} \\
& \times \sum_{\sigma= \pm}\left\{b_{\sigma}(k) \mathcal{U}_{\sigma}(\vec{k}) \mathrm{e}^{-\mathrm{i} k \cdot x}+d_{\sigma}^{+}(k) \mathscr{V}_{\sigma}(\vec{k}) \mathrm{e}^{\mathrm{i} k \cdot x}\right\}, \\
& k=(E, \vec{k}), \quad E=E_{\vec{k}}=\sqrt{\vec{k}^{2}-m^{2}-\mathrm{i} \epsilon}
\end{aligned}
$$

where $b_{\sigma}$ annihilates particles and $d_{\sigma}^{+}$creates antiparticles. The following anticommutators vanish:

$$
\begin{gathered}
\left\{b_{\sigma}(k), b_{\rho}\left(k^{\prime}\right)\right\}=\left\{b_{\sigma}^{+}(k), b_{\rho}^{+}\left(k^{\prime}\right)\right\}=0, \\
\left\{d_{\sigma}(k), d_{\rho}\left(k^{\prime}\right)\right\}=\left\{d_{\sigma}^{+}(k), d_{\rho}^{+}\left(k^{\prime}\right)\right\}=0 .
\end{gathered}
$$

We assume the following general form for the nonvanishing anticommutators,

$$
\begin{aligned}
& \left\{b_{\sigma}(k), b_{\rho}^{+}\left(k^{\prime}\right)\right\}=f(\sigma, \vec{k})(2 \pi)^{3} \frac{E}{m} \delta^{3}\left(\vec{k}-\vec{k}^{\prime}\right) \delta_{\sigma \rho}, \\
& \left\{d_{\sigma}(k), d_{\rho}^{+}\left(k^{\prime}\right)\right\}=g(\sigma, \vec{k})(2 \pi)^{3} \frac{E}{m} \delta^{3}\left(\vec{k}-\vec{k}^{\prime}\right) \delta_{\sigma \rho},
\end{aligned}
$$

with arbitrary $f$ and $g$ functions of the quantum numbers $\sigma$ and $\vec{k}$. One might argue that since $f$ and $g$ must be dimensionless, they can depend only on the dimensionless arguments $\sigma$ and $\vec{k} / m$, but that is a detail of the discussion which we do not pursue any further. Our only assumption concerns the fact that the field anticommutators should be diagonal in the helicity and wave vector quantum numbers, leading to the corresponding Kronecker and Dirac- $\delta$ 's.

We assume that the spin-matrix $\Gamma$ either constitutes a Lorentz scalar or a pseudoscalar quantity, which is a scalar under the proper orthochronous Lorentz group. The timeordered product of field operators reads as

$$
\begin{aligned}
& \langle 0|T \psi(x) \bar{\psi}(y) \Gamma| 0\rangle \\
& =\int \frac{\mathrm{d}^{3} k}{(2 \pi)^{3}} \frac{m}{E} \\
& \quad \times\left\{\Theta\left(x^{0}-y^{0}\right) \mathrm{e}^{-\mathrm{i} k \cdot(x-y)}\right. \\
& \quad \times \sum_{\sigma= \pm} f(\sigma, \vec{k}) \mathcal{U}_{\sigma}(\vec{k}) \otimes \overline{\mathcal{U}}_{\sigma}(\vec{k}) \Gamma \\
& \quad-\Theta\left(y^{0}-x^{0}\right) \mathrm{e}^{\mathrm{i} k \cdot(x-y)} \\
& \left.\quad \times \sum_{\sigma= \pm} g(\sigma, \vec{k}) \mathscr{V}_{\sigma}(\vec{k}) \otimes \overline{\mathscr{V}}_{\sigma}(\vec{k}) \Gamma\right\} .
\end{aligned}
$$


This equation contains the same coefficient functions $f$ and $g$ that enter into (48a) and (48b). In order to proceed with the derivation of the propagator, we must postulate that the following sum rules hold:

$$
\begin{aligned}
& \sum_{\sigma} f(\sigma, \vec{k}) \mathscr{U}_{\sigma}(\vec{k}) \otimes \overline{\mathcal{U}}_{\sigma}(\vec{k}) \Gamma=\frac{k-\gamma^{5} m}{2 m}, \\
& \sum_{\sigma} g(\sigma, \vec{k}) \mathscr{V}_{\sigma}(\vec{k}) \otimes \overline{\mathscr{V}}_{\sigma}(\vec{k}) \Gamma=\frac{k+\gamma^{5} m}{2 m} .
\end{aligned}
$$

The sum rule (50) is crucial for the further steps in the derivation of the time-ordered propagator. Introducing a suitable complex integral representation for the step function, one obtains from (49), using (50), after a few steps which we do not discuss in further detail (see also equations (3.169) and (3.170) of [57])

$$
\begin{aligned}
& \langle 0|T \psi(x) \bar{\psi}(y) \Gamma| 0\rangle \\
& =\mathrm{i} \int \frac{\mathrm{d}^{3} k}{(2 \pi)^{3}} \frac{m}{E} \int \frac{\mathrm{d} k_{0}}{2 \pi} e^{-\mathrm{i} k_{0} \cdot\left(x^{0}-y^{0}\right)+\mathrm{i} \vec{k} \cdot(\vec{x}-\vec{y})} \\
& \quad \times \frac{\gamma^{0} k_{0}-\vec{\gamma} \cdot \vec{k}-\gamma^{5} m}{2 m\left(k_{0}-E+\mathrm{i} \epsilon\right)} \\
& +\mathrm{i} \int \frac{\mathrm{d}^{3} k}{(2 \pi)^{3}} \frac{m}{E} \int \frac{\mathrm{d} k_{0}}{2 \pi} \mathrm{e}^{-\mathrm{i} k_{0} \cdot\left(x^{0}-y^{0}\right)+\mathrm{i} \cdot \vec{k} \cdot(\vec{x}-\vec{y})} . \\
& \times \frac{-\gamma^{0} k_{0}+\vec{\gamma} \cdot \vec{k}+\gamma^{5} m}{2 m\left(k_{0}+E-\mathrm{i} \epsilon\right)} .
\end{aligned}
$$

The convention is that in any integrals $\int \mathrm{d}^{3} k$, the component $k_{0}$ is set equal to $E=\sqrt{\vec{k}^{2}-m^{2}}$ in the integrand when it occurs in scalar products of the form $k \cdot(x-y)$, and so forth, but if the integral is over the full $\mathrm{d}^{4} k$, then the integration interval is the full $k_{0} \in(-\infty, \infty)$. With the convention which is adopted in many quantum field theoretical textbooks, including $[57,60]$, we finally obtain the result

$$
\langle 0|T \psi(x) \bar{\psi}(y) \Gamma| 0\rangle=\mathrm{i} \int \frac{\mathrm{d}^{4} k}{(2 \pi)^{4}} \mathrm{e}^{-\mathrm{i} k \cdot(x-y)} \frac{k-\gamma^{5} m}{k^{2}+m^{2}+\mathrm{i} \epsilon} .
$$

The tachyonic propagator $S_{T}$ is identified, under the integral sign, as

$$
S_{T}(k)=\frac{1}{k-\gamma^{5}(m+\mathrm{i} \epsilon)}=\frac{k-\gamma^{5} m}{k^{2}+m^{2}+\mathrm{i} \epsilon} .
$$

The sum rules (40a) and (40b) imply that the derivation is valid for the choice

$$
f(\sigma, \vec{k})=g(\sigma, \vec{k})=-\sigma, \quad \Gamma=\gamma^{5},
$$

in which case the relations given in (50) are fulfilled. Note that this observation does not imply that the choice (54) necessarily is the only one for which we are able to fulfill the postulates given in (50), but it is the only structurally simple choice that we have found.

For the egregiously simple choice (54), let us study the transition to the massless limit (16) in some further detail. Indeed, in the limit $m \rightarrow 0$, the denominator of the spin sums in (40a) and (40b) vanishes, and a finite limit is obtained after multiplication with $2 m$,

$$
\begin{aligned}
& \lim _{m \rightarrow 0} \sum_{\sigma} 2 m(-\sigma) \mathscr{U}_{\sigma}(\vec{k}) \otimes \overline{\mathscr{U}}_{\sigma}(\vec{k}) \gamma^{5}=k \\
& \lim _{m \rightarrow 0} \sum_{\sigma} 2 m(-\sigma) \mathscr{V}_{\sigma}(\vec{k}) \otimes \overline{\mathscr{V}}_{\sigma}(\vec{k}) \gamma^{5}=k .
\end{aligned}
$$

In order to compare the normalizations of the fundamental spinors in the massless limit, we calculate the following quantities:

$$
\begin{aligned}
\overline{\mathcal{U}}_{\sigma}(\vec{k}) \gamma^{5} \gamma^{0} \mathcal{U}_{\sigma}(\vec{k}) & =-\sigma \frac{E}{m}, \\
\bar{u}_{\sigma}(\vec{k}) \gamma^{5} \gamma^{0} u_{\sigma}(\vec{k}) & =-\sigma, \\
\overline{\mathscr{V}}_{\sigma}(\vec{k}) \gamma^{5} \gamma^{0} \mathscr{V}_{\sigma}(\vec{k}) & =-\sigma \frac{E}{m}, \\
\bar{v}_{\sigma}(\vec{k}) \gamma^{5} \gamma^{0} v_{\sigma}(\vec{k}) & =-\sigma,
\end{aligned}
$$

where the fundamental spinors $u_{\sigma}$ and $v_{\sigma}$ of the massless equation have been given in Section 2.1. Observing that $E=$ $|\vec{k}|$ in the massless limit, the identifications $\sqrt{m} \mathscr{U}_{\sigma}(\vec{k}) \rightarrow$ $\sqrt{|\vec{k}|} u_{\sigma}(\vec{k})$ and $\sqrt{m} \mathscr{V}_{\sigma}(\vec{k}) \rightarrow \sqrt{|\vec{k}|} v_{\sigma}(\vec{k})$, as implied by (56) and (57), show that the identities (55a) and (55b) precisely reduce to the sum rule (16) in the massless limit.

4.2. Generalized Field Anticommutators for Tardyonic and Tachyonic Fields. For definiteness, we have considered the case of the tachyonic Dirac field in the above derivation. The decisive observation is that the tachyonic choice,

tachyonic choice: $f(\sigma, \vec{k})=g(\sigma, \vec{k})=-\sigma, \quad \Gamma=\gamma^{5}$,

is consistent with both massive tachyonic fields discussed in Sections 3.1 and 3.2, whereas the tardyonic choice,

tardyonic choice: $f(\sigma, \vec{k})=g(\sigma, \vec{k})=1, \quad \Gamma=\mathbb{1}_{4 \times 4}$,

yields the time-ordered propagator for both massive tardyonic fields discussed in Sections 2.2 and 2.3. The nonvanishing anticommutators for tardyons take the simple form (cf. (48a) and (48b)),

tardyonic anticommutators:

$$
\begin{aligned}
& \left\{b_{\sigma}(k), b_{\rho}^{+}\left(k^{\prime}\right)\right\}=(2 \pi)^{3} \frac{E}{m} \delta^{3}\left(\vec{k}-\vec{k}^{\prime}\right) \delta_{\sigma \rho}, \\
& \left\{d_{\sigma}(k), d_{\rho}^{+}\left(k^{\prime}\right)\right\}=(2 \pi)^{3} \frac{E}{m} \delta^{3}\left(\vec{k}-\vec{k}^{\prime}\right) \delta_{\sigma \rho} .
\end{aligned}
$$


Again, compared with (48a) and (48b), the nonvanishing anticommutators for tachyons take the simple form,

tachyonic anticommutators:

$$
\begin{aligned}
& \left\{b_{\sigma}(k), b_{\rho}^{+}\left(k^{\prime}\right)\right\}=(-\sigma)(2 \pi)^{3} \frac{E}{m} \delta^{3}\left(\vec{k}-\vec{k}^{\prime}\right) \delta_{\sigma \rho}, \\
& \left\{d_{\sigma}(k), d_{\rho}^{+}\left(k^{\prime}\right)\right\}=(-\sigma)(2 \pi)^{3} \frac{E}{m} \delta^{3}\left(\vec{k}-\vec{k}^{\prime}\right) \delta_{\sigma \rho} .
\end{aligned}
$$

With these universal choices, the theory of the tardyonic and tachyonic spin-1/2 fields can be unified. The time-ordered propagator is given as

$$
\begin{aligned}
& \langle 0|T \psi(x) \bar{\psi}(y) \Gamma| 0\rangle=\mathrm{i} S(x-y), \\
& S(x-y)=\int \frac{\mathrm{d}^{4} k}{(2 \pi)^{4}} \mathrm{e}^{-\mathrm{i} k \cdot(x-y)} S(k) .
\end{aligned}
$$

We use the sum rules for the tensor sums over fundamental spinors given in (25) (for the tardyonic Dirac field), (32) (for the tardyonic Dirac field with two mass terms), (40a) and (40b) (for the tachyonic Dirac field), and (45) (for the Dirac field of imaginary mass). Going through the exact same derivation as outlined above in between (49) and (53), we obtain the following results for the time-ordered propagators of tardyonic and tachyonic fields. For the tardyonic Dirac field (Section 2.2), one has

$$
S^{(1)}(k)=\frac{1}{k-m_{1}+\mathrm{i} \epsilon}=\frac{k+m_{1}}{k^{2}-m_{1}^{2}+\mathrm{i} \epsilon} .
$$

For the tardyonic field with two mass terms (Section 2.3), the Feynman propagator is easily found as

$$
\begin{aligned}
S^{(t)}(k) & =\frac{1}{k-m_{1}+\mathrm{i} \epsilon-\mathrm{i} \gamma^{5}\left(m_{2}-i \eta\right)} \\
& =\frac{k+m_{1}-\mathrm{i} \gamma^{5} m_{2}}{k^{2}-m_{1}^{2}-m_{2}^{2}+\mathrm{i} \epsilon},
\end{aligned}
$$

where $\epsilon$ and $\eta$ are infinitesimal imaginary parts. Both tardyonic mass terms acquire an infinitesimal negative imaginary part, and the prefactor $m / E$ from (46) in the field operator needs to be replaced by $m_{1} / E^{(t)}$, where the tardyonic energy is $E^{(t)}=\sqrt{\vec{k}^{2}+m_{1}^{2}+m_{2}^{2}}$. For the tachyonic Dirac field (Section 3.1 in [9]), one has the result given in (53). Finally, for the Dirac field with two tachyonic mass terms, we have

$$
S^{\prime}(k)=\frac{k+\mathrm{i} m_{1}-\gamma^{5} m_{2}}{k^{2}+m_{1}^{2}+m_{2}^{2}+\mathrm{i} \epsilon} .
$$

In the latter case, the prefactor $m / E$ from (46) in the field operator needs to be replaced by $m_{1} / E^{\prime}$, where the tachyonic energy is $E^{\prime}=\sqrt{\vec{k}^{2}-m_{1}^{2}-m_{2}^{2}}$. For both tachyonic fields discussed here, the mass acquires an infinitesimal positive imaginary part, as manifest in the results given in (53) and (65).
4.3. Tachyonic Gordon Identities. It is useful to illustrate the derivation outlined above by exploring its connection to tachyonic Gordon identities. For definiteness, we again concentrate on the tachyonic Dirac equation discussed in Section 3.1. The matrix element of the vector current finds the following Gordon decomposition for positive-energy spinors:

$$
\begin{aligned}
& \overline{\mathscr{U}}_{ \pm}\left(\vec{k}^{\prime}\right) \gamma^{\mu} \mathscr{U}_{ \pm}\left(\vec{k}^{\prime}\right) \\
& \quad=\frac{1}{2 m} \overline{\mathscr{U}}_{ \pm}\left(\vec{k}^{\prime}\right) \gamma^{5}\left[\left(k^{\prime \mu}-k^{\mu}\right)+\mathrm{i} \sigma^{\mu \nu}\left(k_{v}^{\prime}+k_{v}\right)\right] \mathscr{U}_{ \pm}(\vec{k}) .
\end{aligned}
$$

For negative-energy solutions, the identity reads as

$$
\begin{aligned}
& \overline{\mathscr{V}}_{ \pm}\left(\vec{k}^{\prime}\right) \gamma^{\mu} \mathscr{V}_{ \pm}(\vec{k}) \\
& \quad=-\frac{1}{2 m} \overline{\mathscr{V}}_{ \pm}\left(\vec{k}^{\prime}\right) \gamma^{5}\left[\left(k^{\prime \mu}-k^{\mu}\right)+\mathrm{i} \sigma^{\mu \nu}\left(k_{v}^{\prime}+k_{v}\right)\right] \mathscr{V}_{ \pm}(\vec{k}) .
\end{aligned}
$$

For $k^{\prime}=k$, one has

$$
\begin{gathered}
\overline{\mathscr{U}}_{ \pm}(\vec{k}) \gamma^{\mu} \mathscr{U}_{ \pm}(\vec{k})=\frac{\mathrm{i}}{m} \overline{\mathscr{U}}_{ \pm}(\vec{k}) \gamma^{5} \sigma^{\mu \nu} k_{v} \mathscr{U}_{ \pm}(\vec{k}) \\
\overline{\mathscr{V}}_{ \pm}(\vec{k}) \gamma^{\mu} \mathscr{V}_{ \pm}(\vec{k})=-\frac{\mathrm{i}}{m} \overline{\mathscr{V}}_{ \pm}(\vec{k}) \gamma^{5} \sigma^{\mu \nu} k_{v} \mathscr{V}_{ \pm}(\vec{k})
\end{gathered}
$$

The matrix element of the axial current reads

$$
\begin{aligned}
& \overline{\mathscr{U}}_{ \pm}\left(\vec{k}^{\prime}\right) \gamma^{5} \gamma^{\mu} \mathscr{U}_{ \pm}(\vec{k}) \\
& \quad=-\frac{1}{2 m} \overline{\mathscr{U}}_{ \pm}\left(\vec{k}^{\prime}\right)\left[\left(k^{\prime \mu}+k^{\mu}\right)+\mathrm{i} \sigma^{\mu \nu}\left(k_{v}^{\prime}-k_{v}\right)\right] \mathscr{U}_{ \pm}(\vec{k}),
\end{aligned}
$$

whereas for negative-energy solutions

$$
\begin{aligned}
& \overline{\mathscr{V}}_{ \pm}\left(\vec{k}^{\prime}\right) \gamma^{5} \gamma^{\mu} \mathscr{V}_{ \pm}(\vec{k}) \\
& \quad=\frac{1}{2 m} \overline{\mathscr{V}}_{ \pm}\left(\vec{k}^{\prime}\right)\left[\left(k^{\prime \mu}+k^{\mu}\right)+\mathrm{i} \sigma^{\mu \nu}\left(k_{v}^{\prime}-k_{v}\right)\right] \mathscr{V}_{ \pm}(\vec{k})
\end{aligned}
$$

For $k^{\prime}=k$, this simplifies to

$$
\begin{aligned}
& \overline{\mathscr{U}}_{ \pm}(\vec{k}) \gamma^{5} \gamma^{\mu} \mathscr{U}_{ \pm}(\vec{k})=-\frac{1}{m} \overline{\mathscr{U}}_{ \pm}(\vec{k}) k^{\mu} \mathscr{U}_{ \pm}(\vec{k}), \\
& \overline{\mathscr{V}}_{ \pm}(\vec{k}) \gamma^{5} \gamma^{\mu} \mathscr{V}_{ \pm}(\vec{k})=\frac{1}{m} \overline{\mathscr{V}}_{ \pm}(\vec{k}) k^{\mu} \mathscr{V}_{ \pm}(\vec{k}) .
\end{aligned}
$$

The results (69a), (69b), (70a), and (70b) for the tachyonic axial vector current have a similar structure as the Gordon decomposition for the tardyonic vector current obtained with the ordinary Dirac equation (see equation (2.54) of [57]). The role of the Dirac adjoint for the tardyonic case is taken over by the "chiral adjoint" $\overline{\mathcal{U}}_{ \pm}(\vec{k}) \gamma^{5}$ for the tachyonic particle. Here, the designation "chiral adjoint" is inspired by the fact that 
$\bar{U}_{ \pm}(\vec{k}) \gamma^{5} \mathcal{U}_{ \pm}(\vec{k})$ transforms as a pseudoscalar under Lorentz transformations.

The structure of (68a), (68b), (70a), and (70b) is somewhat peculiar with regard to parity. In (68a), an apparent vector current on the left-hand side appears to transform into an axial current on the right-hand side, whereas in (70a), an apparent axial vector on the left-hand side of the equation becomes what appears to be a vector on the right-hand side. The reason lies in the more complicated behavior of the tachyonic Dirac equation under parity as investigated in [10]. Namely, the tachyonic Dirac equation (7) contains a term which transforms as a scalar under parity,

$$
\mathrm{i} \gamma^{\nu} \partial_{\mu} \stackrel{\mathscr{P}}{\longrightarrow} \gamma^{0}\left(\mathrm{i} \gamma^{0} \partial_{0}+\mathrm{i} \gamma^{i}\left(-\partial_{i}\right)\right) \gamma^{0}=\mathrm{i} \gamma^{\nu} \partial_{\mu},
$$

as well as a term which transforms as a pseudoscalar,

$$
\gamma^{5} m \stackrel{\mathscr{P}}{\longrightarrow} \gamma^{0}\left(\gamma^{5} m\right) \gamma^{0}=-\gamma^{5} m .
$$

The mass term in the tachyonic Dirac equation is pseudoscalar and changes sign under parity. Indeed, in [10], the tachyonic Dirac equation has been shown to be separately $\mathscr{C} \mathscr{P}$ invariant, and $\mathscr{T}$ invariant, but not $\mathscr{P}$ invariant, due to the change in the mass term.

In order to put this observation into perspective, we recall that the entries of the electromagnetic field strength tensor are composed of axial vector components (magnetic $\vec{B}$ field), as well as vector components (electric $\vec{E}$ field). The transformation properties of the electromagnetic field strength tensor under the proper orthochronous Lorentz group are nevertheless well defined.

The transformation (71b) can be interpreted as a transformation $m \rightarrow-m$ under parity. Thus, if we interpret the mass $m$ as a pseudoscalar quantity, then the right-hand sides of (68a) and (70a) transform as a vector and an axial vector, respectively. It is the parity noninvariance of the mass term in the tachyonic Dirac equation which leads to the somewhat peculiar structure of (68a) and (70a).

4.4. Helicity-Dependence and Gupta-Bleuler Condition. The anticommutator relations for tardyons given in (60) imply that both left-handed and right-handed helicity states, for both particles and antiparticles, have positive norm. However, the anticommutator relations for tachyons given in (61) imply that right-handed particle as well as left-handed antiparticle states acquire negative norm. This is shown in equations (31) and (32) of [9]. Indeed, for one-particle states $\left|1_{k, \sigma}\right\rangle=b_{\sigma}^{+}(k)|0\rangle$,

$$
\begin{aligned}
\left\langle 1_{k, \sigma} \mid 1_{k, \sigma}\right\rangle & =\left\langle 0\left|b_{\sigma}(k) b_{\sigma}^{+}(k)\right| 0\right\rangle \\
& =\left\langle 0\left|\left\{b_{\sigma}(k), b_{\sigma}^{+}(k)\right\}\right| 0\right\rangle \\
& =(-\sigma) V \frac{E}{m},
\end{aligned}
$$

where $V=(2 \pi)^{3} \delta^{3}(\overrightarrow{0})$ is the normalization volume in coordinate space. The Fock-space norm $\left\langle 1_{k, \sigma} \mid 1_{k, \sigma}\right\rangle$ is negative for $\sigma=1$.
For clarification, the corresponding Gupta-Bleuler condition should be indicated explicitly. In full analogy to the instructive discussion of the Gupta-Bleuler mechanism for the photon field, as given in full clarity in Chapter (9b) of Schweber's textbook [61], we select the positive- and negativefrequency component of the $(\sigma=1)$-component of the neutrino field operator,

$$
\begin{aligned}
& \psi_{\sigma=1}^{(+)}(x)=\int \frac{\mathrm{d}^{3} k}{(2 \pi)^{3}} \frac{m}{E} b_{\sigma=1}(k) \mathscr{U}_{\sigma=1}(\vec{k}) \mathrm{e}^{-\mathrm{i} k \cdot x}, \\
& \psi_{\sigma=1}^{(-)}(x)=\int \frac{\mathrm{d}^{3} k}{(2 \pi)^{3}} \frac{m}{E} d_{\sigma=1}^{+}(k) \mathscr{V}_{\sigma=1}(\vec{k}) \mathrm{e}^{\mathrm{i} k \cdot x},
\end{aligned}
$$

and postulate that it annihilates any physical Fock state $|\Psi\rangle$ of the tachyonic field,

$$
\left\langle\Psi\left|\psi_{\sigma=1}^{(-)}(x)=\psi_{\sigma=1}^{(+)}(x)\right| \Psi\right\rangle=0 .
$$

These relations automatically imply that the Gupta-Bleuler condition also is realized in terms of the expectation value

$$
\left\langle\Psi\left|\psi_{\sigma=1}(x)\right| \Psi\right\rangle=\left\langle\Psi\left|\psi_{\sigma=1}^{(-)}(x)+\psi_{\sigma=1}^{(+)}(x)\right| \Psi\right\rangle=0,
$$

but the condition (74) is stronger. We recall that the GuptaBleuler condition on the photon field reads $\left\langle\Psi_{\gamma}\left|\partial^{\mu} A_{\mu}\right| \Psi_{\gamma}\right\rangle=$ 0 , where $\left|\Psi_{\gamma}\right\rangle$ is a Fock state of the photon field. As stressed in Schweber's book [61] on page 246, the condition $\left\langle\Psi_{\gamma}\left|\partial_{\mu} A^{\mu}\right| \Psi_{\gamma}\right\rangle=0$ is not sufficient for the suppression of the longitudinal and scalar photons, but one must postulate that $\partial^{\mu} A_{\mu}^{(+)}\left|\Psi_{\gamma}\right\rangle=0$, where $A_{\mu}^{(+)}$is the positive-frequency component of the photon field operator. Because the tachyonic fermion, unlike the photon, is not equal to its own antiparticle, we need two conditions, given in (74).

A crucial question now concerns the possibility of reversing the helicity-dependence, that is, the question of whether or not a different choice for the helicity-dependent factors in (48a) and (48b) exists that would imply negative norm for left-handed particles and right-handed antiparticles. A related question is whether other tachyonic Dirac Hamiltonians exist for which the choice $f(\sigma, \vec{k})=g(\sigma, \vec{k})=+\sigma$ instead of $(-\sigma)$ would fulfill our general postulate, namely, the sum rule (50). For reasons outlined in the following, we can ascertain that this is not the case; tachyonic spin- $1 / 2$ particles should always be left handed.

The arguments supporting this conclusion are as follows. First, if we assume that the tachyonic field fulfills a sum rule of type II which for massless fields is given in (16), then it is impossible to replace $(-\sigma)$ by $(+\sigma)$ in the sum rule because of the necessity to preserve a smooth massless limit. The considerations in the text following (16) imply that if one were to replace $(-\sigma)$ by $(+\sigma)$ in the massless case, then one would violate the sum rule (16). The second argument is obtained by explicit calculation. We have checked that if one replaces $m \rightarrow-m$ in the tachyonic and imaginary-mass Dirac equations (7) and (9), then the sum rules fulfilled by the corresponding fundamental spinors still contain the characteristic factor $(-\sigma)$. For the imaginarymass Dirac equation, this result is obtained in [25]. Intuitively, 
we can understand this result as follows: the mass $m$ in the denominators of the right-hand sides of (40a), (40b), and (45) is obtained as the modulus $\sqrt{m^{2}}=|m|$ and does not change if we replace $m \rightarrow-m$ in the superluminal Dirac equation. The mass in the numerator of the right-hand sides of (40a), (40b), and (45) changes sign, but this is consistent with the obvious change in the functional form of the positive-energy and negative-energy projectors as we change the sign of the mass term. Again, this consideration supports the conclusion that we cannot invert the helicity-dependence by choosing a different Hamiltonian; the factor $(-\sigma)$ persists.

The third argument comes from the tachyonic Gordon identities discussed in Section 4.3. We use the Gordon identity (70a) and (70b) and the normalization (39) to calculate the bispinor trace (with a $\gamma^{0}$ multiplied from the right) of the left-hand side of (40a),

$$
\begin{aligned}
& \operatorname{tr}\left(\sum_{\sigma}(-\sigma) \mathcal{U}_{\sigma}(\vec{k}) \otimes \overline{\mathcal{U}}_{\sigma}(\vec{k}) \gamma^{5} \gamma^{0}\right) \\
& =\sum_{\sigma}(-\sigma) \overline{\mathcal{U}}_{\sigma}(\vec{k}) \gamma^{5} \gamma^{\mu=0} \mathcal{U}_{\sigma}(\vec{k}) \\
& =\sum_{\sigma}(-\sigma)\left(-\frac{k^{0}}{m}\right) \underbrace{\overline{\boldsymbol{U}}_{\sigma}(\vec{k}) \mathcal{U}_{\sigma}(\vec{k})}_{=\sigma}=2 \frac{E}{m} .
\end{aligned}
$$

The bispinor trace of the right-hand side of (40a) is

$$
\operatorname{tr}\left(\gamma^{0} \frac{k-\gamma^{5} m}{2 m}\right)=\operatorname{tr}\left(\gamma^{0} \frac{k}{2 m}\right)=4 \frac{E}{2 m}=2 \frac{E}{m},
$$

which shows the consistency of the bispinor sum (40a) with the Gordon decomposition (70a) and (70b). If we were to replace $(-\sigma)$ by $(+\sigma)$, the two sides of the relation (76a) would differ by a minus sign. The bispinor trace of the left-hand side of $(40 \mathrm{~b})$ is

$$
\begin{aligned}
& \operatorname{tr}\left(\sum_{\sigma}(-\sigma) \mathscr{V}_{\sigma}(\vec{k}) \otimes \overline{\mathscr{V}}_{\sigma}(\vec{k}) \gamma^{5} \gamma^{0}\right) \\
& =\sum_{\sigma}(-\sigma) \overline{\mathscr{V}}_{\sigma}(\vec{k}) \gamma^{5} \gamma^{\mu=0} \mathscr{V}_{\sigma}(\vec{k}) \\
& =\sum_{\sigma}(-\sigma)\left(\frac{k^{0}}{m}\right) \underbrace{\overline{\mathscr{V}}_{\sigma}(\vec{k}) \mathscr{V}_{\sigma}(\vec{k})}_{=-\sigma}=2 \frac{E}{m} .
\end{aligned}
$$

We have used the tachyonic Gordon decomposition for negative-energy states as given in (70a) and (70b), which differs from the positive-energy Gordon decomposition by a minus sign, but an additional minus sign is obtained from the Lorentz-invariant normalization of the negative-energy fundamental bispinors. From the right-hand side of (40b), we have

$$
\operatorname{tr}\left(\gamma^{0} \frac{k+\gamma^{5} m}{2 m}\right)=\operatorname{tr}\left(\gamma^{0} \frac{k}{2 m}\right)=2 \frac{E}{m},
$$

which again is fully consistent, but only because we have a factor $(-\sigma)$ in the sum rule (40a) and (40b), which combines with the factor $(-\sigma)$ from the Lorentz-invariant normalization of the $\overline{\mathscr{V}}_{\sigma}(\vec{k}) \mathscr{V}_{\sigma}(\vec{k})$, to give $2 E / m$ as a final result in (77a) and (77b).

\section{Physical Interpretation: From Tachyonic Neutrinos to Cosmology}

5.1. Arguments for and against Tachyonic Neutrinos. In the absence of conclusive experimental evidence, the hypothesis of tachyonic neutrinos has been controversially discussed in the literature. Three main arguments [62] have been brought forward against tachyonic neutrinos. (1) They would require us to give up the notion of a Lorentz-invariant vacuum state, and even the vacuum would become unstable in the presence of tachyonic fields. (2) Given the tachyonic dispersion relation $E=\sqrt{\vec{k}^{2}-m^{2}}$, the role of states with $|\vec{k}|<m$ needs to be clarified. (3) The physical (probability!?) interpretation of the conserved Noether current of the free tachyonic Dirac equation has been called into question [62], and it has been argued that no consistent interpretation can be given because certain zero components of the conserved current were conjectured to vanish for all tachyonic momentum eigenstates [62].

A possible answer for question (1) has been proposed in [9]. Summarizing the argument, it has been concluded in [9] that one can solve the problem in two ways. (i) One can Lorentz transform the vacuum state and Lorentz transform all fundamental creation and annihilation operators of the fermion field (some of these will change from annihilators to creators upon transformation, due to the spacelike nature of the tachyons). (ii) One keeps a Lorentz-invariant vacuum state, and only transform the space-time arguments $k^{\mu}$ and $x^{\mu}$ of the field operators, keeping all creation operators as creators and annihilation operators as annihilators. The amplitudes, cross sections, and so forth, obtained using approach (ii), then depend on scalar product of four vectors which are equal to the result obtained by first calculating the process in the original Lorentz frame and then performing the Lorentz transformation into the moving frame.

The conjecture regarding an expansion of a "false" vacuum in the presence of tachyons can be traced to the fact that most of the tachyonic theories discussed so far in the literature are scalar [28-33]. Indeed, scalar tachyons have a problem with instability, because of the structure of the mass term in relation to the field Hamiltonians, which changes sign $m^{2} \rightarrow-m^{2}$ in a tachyonic theory, suggesting that the field energy can be lowered by creating tachyons. The problem does not occur in tachyonic spin-1/2 theories because a linear, not quadratic, mass term enters the field Lagrangian and Hamiltonian. Provided one reinterprets the spin-1/2 antiparticle solutions in the usual way (negative energy for propagation into the past becomes positive energy for propagation into the future), it then becomes immediately clear that the vacuum energy cannot be lowered upon spin$1 / 2$ tachyon antitachyon pair production.

A solution to problem (2) has also been proposed in [9]. Namely, the energies with $E= \pm \sqrt{\vec{k}^{2}-m^{2}-\mathrm{i} \epsilon}($ for $|\vec{k}|<m)$ find a natural interpretation in terms of complex resonance and antiresonance energies, which describe unstable states which decay in time. Particle resonances are damped for propagation into the future, and antiparticle antiresonances 
are damped for propagation into the past, as they should be [9]. The occurrence of momentum eigenstates with real energies, and resonances with complex resonance energies, is a well-known phenomenon all across physics (e.g., in atomic physics, the ground-state energy of the helium atom is strictly real, whereas autoionizing resonances in the three-particle system have a manifestly complex resonance energy).

Another "myth" which should be refuted concerns a conceivable "runaway reaction" where a moving tachyon releases an arbitrarily large amount of energy, as it loses energy and accelerates, given its classical energy-velocity relation $E=m / \sqrt{v^{2}-1}$. According to this relation, a tachyon indeed accelerates as its energy is lowered and becomes commensurate with the invariant mass square. (At high energy, a tachyon approaches the light cone, though.) An infinitely fast tachyon takes the role of a tardyon at rest [28]; the explicit eigenstates have been indicated in [9]. However, energy conservation holds, and one cannot mix the notion of energy increase by acceleration, which only holds for tardyonic particles, in order to "convert" a tachyon losing energy by acceleration into a tardyon that gains energy in the same process. The "runaway reaction" is impossible; only a finite amount of energy is released as the tachyon accelerates and the energy goes from $E=m / \sqrt{v^{2}-1}$ to zero. Energy conservation holds for tachyons, even if they accelerate, somewhat counterintuitively, when losing energy.

An answer to question (3) has not yet been provided so far in the literature to the best of our knowledge. Here, we aim to provide a possible physical interpretation for the conserved current and scalar product and also show where certain arguments presented originally by Hughes and Stephenson in their research paper [62] entitled "against tachyonic neutrinos" become inconsistent. The Lagrangian density of the tachyonic Dirac particle reads, in first quantization [6],

$$
\mathscr{L}(x)=\bar{\psi}(x) \gamma^{5}\left(\mathrm{i} \gamma^{\mu} \partial_{\mu}-\gamma^{5} m\right) \psi(x) .
$$

This is equivalent, up to partial integration, to the symmetric form [10]

$$
\mathscr{L}=\frac{\mathrm{i}}{2}\left(\bar{\psi} \gamma^{5} \gamma^{\mu}\left(\partial_{\mu} \psi\right)-\left(\partial_{\mu} \bar{\psi}\right) \gamma^{5} \gamma^{\mu} \psi\right)-m \bar{\psi} \psi,
$$

where we suppress the space-time argument $x=(t, \vec{r})$. The nonsymmetric form (78) clearly exhibits the presence of the "chiral adjoint" $\bar{\psi}(x) \gamma^{5}$ in the Lagrangian. The conserved current is

$$
\mathscr{f}^{\mu}(x)=\bar{\psi}(x) \gamma^{5} \gamma^{\mu} \psi(x), \quad \partial_{\mu} \mathcal{F}^{\mu}(x)=0 .
$$

The zero component

$$
\mathscr{J}^{0}(x)=\bar{\psi}(x) \gamma^{5} \gamma^{0} \psi(x)
$$

assumes the following values in plane-wave eigenstates, according to (56) and (57):

$$
\begin{aligned}
\overline{\mathscr{U}}_{\sigma}(\vec{k}) \gamma^{5} \gamma^{0} \mathscr{U}_{\sigma}(\vec{k}) & =-\sigma \frac{E}{m}, \\
\overline{\mathscr{V}}_{\sigma}(\vec{k}) \gamma^{5} \gamma^{0} \mathscr{V}_{\sigma}(\vec{k}) & =-\sigma \frac{E}{m} .
\end{aligned}
$$

We conclude that $\mathscr{J}^{0}$ is positive for left-handed particle states $(\sigma=-1)$ and right-handed for antiparticle states (likewise, $\sigma=-1$; the helicity is equal to $-\sigma$ for antiparticles). For $E=0$, the "axial norm" of the momentum eigenstates given in (82a) and $(82 \mathrm{~b})$ vanishes, but this is a very special case. Namely, for tachyons, $E=0$ implies $|\vec{k}|=m=m v / \sqrt{v^{2}-1}$ for the momentum and thus corresponds to an "infinitely fast" tachyon $(v \rightarrow \infty)$, which remains "infinitely fast" upon Lorentz transformation. It would thus be wrong to conclude, as done in [62] in the text following (54) of [62], that all tachyonic states have zero axial norm, because this would correspond to a forbidden generalization of a result which holds asymptotically, for $v \rightarrow \infty$, to all finite values of the tachyonic velocity $v$. Our (82a) and (82b) gives the explicit result for any finite value of the energy $E$.

The spatial integral of the zero component of the conserved current,

$$
\begin{aligned}
\int \mathrm{d}^{3} r \mathcal{F}^{0}(x) & =\int \mathrm{d}^{3} r \bar{\psi}(x) \gamma^{5} \gamma^{\mu} \psi(x) \\
& =-\int \mathrm{d}^{3} r \psi^{+}(x) \gamma^{5} \psi(x),
\end{aligned}
$$

is precisely equal (up to a sign) to the scalar product $\left\langle\psi_{1}(t), \psi_{2}(t)\right\rangle \equiv \int \mathrm{d}^{3} r \psi_{1}^{+}(t, \vec{r}) \gamma^{5} \psi_{2}(t, \vec{r})$ which is conserved under the time-evolution by the $\gamma^{5}$ Hermitian (pseudoHermitian) Hamiltonian $H_{5}$. This scalar product is not positive definite, as already noticed in the work of Pauli [13], and precisely corresponds to the scalar product introduced by Pauli in equation (3) of [13], where in the notation of [13] we have $\eta=\gamma^{5}$. Similar observations have been made in equations (34) and (52) of [62]. According to [63, 64], one could otherwise define a so-called $\mathscr{C}$ operator, which is not equal to the charge conjugation operator and "remedies" the problem of negative norm attained by some states under the $\gamma^{5}$ norm, leading to a redefined, positivedefinite scalar product. However, the negative norm finds a rather natural interpretation, and a redefinition of the scalar product $\left\langle\psi_{1}(t), \psi_{2}(t)\right\rangle \equiv \int \mathrm{d}^{3} r \psi_{1}^{+}(t, \vec{r}) \gamma^{5} \psi_{2}(t, \vec{r})$ therefore is not required.

For the ordinary Dirac equation, the conserved current is $J^{\mu}(x)=\bar{\psi}_{e}(x) \gamma^{\mu} \psi_{e}(x)$. Its timelike component is $J^{0}(x)=$ $\psi_{e}^{+}(x) \psi_{e}(x)$, where the subscript $e$ reminds us of the electron. The latter can be interpreted as a positive-definite probability density which is conserved under the time evolution generated by the ordinary Hermitian Dirac Hamiltonian $H^{(1)}$.

The tachyonic Dirac current $\mathcal{g}^{\mu}$ is obtained from the ordinary Dirac $J^{\mu}$ by the replacement $\bar{\psi}_{e}(x) \rightarrow \bar{\psi}(x) \gamma^{5}$. We have seen that the scalar product for the tachyonic Dirac Hamiltonian is equal to an integral over the timelike component of the conserved Noether current of the Dirac equation and is not positive definite. In order to put this observation into perspective, it is instructive to recall that for the Klein-Gordon equation $\left(\partial_{\mu} \partial^{\mu}+m^{2}\right) \phi(x)=0$, the zero component of the conserved current $j^{\mu}(x)=$ $(\mathrm{i} / 2 m)\left(\phi^{*}(x) \partial_{\mu} \phi(x)-\phi(x) \partial^{\mu} \phi^{*}(x)\right)$ is not positive definite, either. Therefore, the zero component of the Klein-Gordon current cannot be interpreted as a probability density but 
must be interpreted as a charge density, which is positive for particles and negative for antiparticles.

This interpretation is not available for the zero component of the Noether current of the tachyonic Dirac equation, because the equation is not charge conjugation invariant and is primarily proposed to describe neutrinos [10]. However, we can come closer to a physical interpretation of the timelike component of the Noether current of the tachyonic equation if we compare the interaction Lagrangian $\mathscr{L}_{\mathrm{QED}}$ of quantum electrodynamics to the weak interaction $\mathscr{L}_{W}$ of a neutrino and a "heavy photon," that is, a $Z^{0}$ boson,

$$
\begin{aligned}
\mathscr{L}_{\mathrm{QED}} & =-e \bar{\psi}_{e} \gamma^{\mu} \psi_{e} A_{\mu} \\
\longleftrightarrow \mathscr{L}_{W} & =-\frac{e}{2 \sin \theta_{W} \cos \theta_{W}} \bar{\psi}\left(\gamma^{\mu} \frac{1-\gamma^{5}}{2}\right) \psi Z_{\mu},
\end{aligned}
$$

where $\theta_{W}$ is the Weinberg angle. The axial vector part $\mathscr{L}_{W}^{A}$ of $\mathscr{L}_{W}$ is

$$
\mathscr{L}_{W}^{A}=-\frac{e}{2 \sin \left(2 \theta_{W}\right)} \bar{\psi} \gamma^{5} \gamma^{\mu} \psi Z_{\mu}
$$

where the ordering of the $\gamma$ matrices is important. For the interaction with the timelike component of the vector potential, we have the expressions

$$
\begin{aligned}
\mathscr{L}_{\mathrm{QED}}^{0} & =-e \bar{\psi}_{e} \gamma^{0} \psi_{e} A_{0} \\
\longleftrightarrow \mathscr{L}_{W}^{A, 0} & =-\frac{e}{2 \sin \left(2 \theta_{W}\right)} \bar{\psi} \gamma^{5} \gamma^{0} \psi Z_{0} .
\end{aligned}
$$

For quantum electrodynamics (QED), we interpret $\bar{\psi}_{e} \gamma^{0} \psi_{e}=$ $\psi_{e}^{+} \psi_{e}$ as the probability density, and this suggests an interpretation of the expression $\bar{\psi} \gamma^{5} \gamma^{0} \psi$ as an "axial probability density" or "axial interaction density" of the neutrino field with the timelike component of the $Z^{0}$ boson. According to (82a) and (82b), the "axial interaction density" is positive for the physically allowed states (left-handed particle and right-handed antiparticle states) and negative for the physically forbidden states (right-handed particle and left-handed antiparticle states). This consideration is independent of the suppression mechanism for the states of "wrong" helicity which, in second quantization, proceeds due to negative norm (see the discussion following (72)).

In general, the physical interpretation of tachyonic theories has been discussed by Feinberg $[32,33]$ and Saudarshan et al. [28-31]. The reinterpretation principle is a cornerstone of the theory. The only physically sensible quantities in a quantum theory are transition amplitudes. If the time ordering of superluminal events changes upon Lorentz transformation, then one reinterprets the amplitude as connecting two space-time events whose coordinates are transformed according to the Lorentz transformation, so that the only physically sensible quantity (transition amplitude) simply connects two events, one of which happens before the other [31]. This may seem counterintuitive at first, but it is perhaps a little less counterintuitive if we take into account that the accepted formulation of quantum field theory is based on the Feynman propagator and on the reinterpretation principle for the "advanced" part of the Feynman Green function, which propagates antiparticle solutions into the past. In order to avoid problems with regard to causality, the canonical quantum field theory of subluminal particles has to be supplemented by a reinterpretation principle, just like the tachyonic theory. The physical interpretation of the Noether current is not affected by this consideration.

Finally, let us point out that even without invoking reinterpretation, superluminal propagation can be compatible with causality if we postulate that the tachyonic mass $m$ is so small that the superluminality is within the limits set forth by the uncertainty relation. With an energy $E=m / \sqrt{\beta^{2}-1} \approx$ $m / \sqrt{2 \delta}$ with $v=1+\delta$ and $\delta \ll 1$, we have $\Delta E \Delta t \approx$ $m / \sqrt{2 \delta} \Delta t \leq 1$ (in units with $\hbar=1$ ). Thus, for an infinitesimal tachyonic mass parameter which does not exceed $m \leq$ $\sqrt{2 \delta} / \Delta t$, causality is preserved within the limits set by the uncertainty principle. Quantum limitations, the role of unstable modes and quantum tunneling in superluminal propagation have been discussed in [65-68].

5.2. Gupta-Bleuler Condition and Seesaw Mechanism. The commonly accepted mechanism for the suppression of righthanded neutrino and left-handed antineutrino states is the seesaw mechanism [69]. After integrating the heavy degrees of freedom (the sterile neutrino), it contains a nonrenormalizable dimension five operator and has a hierarchy problem: Namely, the neutrino masses are inversely proportional to the grand unification (GUT) scale $\Lambda$ and sensitively depend on fine-tuning of $\Lambda$. Let us consider equation (18) of [69] and hypothetically consider a formulation that would result if the physically observable neutrino were right handed. Then, we could reformulate equation (18) of [69] as

$$
\mathscr{L}_{I}^{\text {eff }}=-\frac{1}{\Lambda} \sum_{r, r^{\prime}}\left[\bar{R}_{r^{\prime} R} \widetilde{H}\right] Y_{r^{\prime} r}\left[\widetilde{H}^{T}\left(R_{r R}\right)^{c}\right]+\text { h.c. }
$$

where

$$
R_{r R}=\left(\begin{array}{c}
v_{r R} \\
r_{R}
\end{array}\right), \quad H=\left(\begin{array}{c}
H^{(+)} \\
H^{(0)}
\end{array}\right) .
$$

With the expectation value of the Higgs field,

$$
\widetilde{H}_{0}=\frac{1}{\sqrt{2}}\left(\begin{array}{l}
v \\
0
\end{array}\right)
$$

instead of the left-handed Majorana neutrino masses, the right-handed ones would be small,

$$
\begin{gathered}
\mathscr{L}^{M}=-\frac{1}{2} \sum_{r, r^{\prime}} \bar{v}_{r^{\prime} R} M_{r r^{\prime}}^{R}\left(v_{r R}\right)^{c}+\text { h.c. } \\
M_{r r^{\prime}}^{R}=\frac{v^{2}}{\Lambda} Y_{r^{\prime} r} .
\end{gathered}
$$

The seesaw mechanism is not unique in suppressing a definite helicity of the neutrino. It is unique provided one formulates it in terms of the left-handed fermion fields, but it could be formulated with inverted helicities if the helicity of 
the observed neutrinos at low energy were different. In the latter case, the right-handed neutrino mass would be small, instead of the left-handed one. On the other hand, the seesaw mechanism has the distinct advantage that it is not necessary to assume a superluminal character of the neutrino.

The mechanism discussed here in the text following (72) is definite in making a prediction regarding the suppression of right-handed neutrino states, as explained by three independent arguments in Section 4.4. However, we have to assume a superluminal neutrino. While an interacting superluminal field theory is problematic, it is perhaps not as problematic as previously thought (see [70] and Section 4 of [9]).

Final clarification can only come from experiment. The seesaw mechanism is compatible with a Majorana neutrino. The tachyonic Dirac equation implies that the neutrino cannot be equal to its antiparticle, because it does not allow charge conjugation invariant solutions. It is only $\mathscr{C} \mathscr{P}$ but not $\mathscr{C}$ invariant. Experimental evidence for neutrinoless double beta decay is disputed [71], and direct measurements of the neutrino mass square currently exclude neither positive nor negative values [44-50]. The generally accepted seesaw mechanism implies that neutrino masses are generated by a nonrenormalizable interaction with a concomitant hierarchy problem, and the mechanism in itself could be reformulated with opposite helicities. It is compatible with a Majorana neutrino. By contrast, a tachyonic neutrino is "automatically" left handed and not equal to its own antiparticle. It is described by a $\gamma^{5}$ Hermitian Hamiltonian and is plagued with the conceptual difficulties associated with (ever so slightly) superluminal propagation. This means that it is experimentally possible to test the models.

5.3. Tachyonic Neutrinos as a Candidate for Dark Energy. The formulation of a gravitational interaction of a spin- $1 / 2$ particle is nontrivial in the quantized formalism. Brill and Wheeler [72] performed the pioneering steps in this direction. In order to formulate the gravitational coupling of a Dirac particle, one has to formulate generalized Dirac matrices $\bar{\gamma}^{\mu}$, which fulfill anticommutation relations compatible with the local metric $\bar{g}^{\mu v}(x)$ of curved space-time. Based on the Christoffel symbols $\Gamma_{\mu \nu}^{\rho}=\Gamma_{\mu \nu}^{\rho}(x)$, one formulates the Christoffel affine connection matrices $\Gamma_{\mu}$ in spinor space and calculates the covariant derivative $\nabla_{\mu}$ as follows [72-76]:

$$
\begin{gathered}
\left\{\bar{\gamma}^{\mu}(x), \bar{\gamma}^{\mu}(x)\right\}=2 \bar{g}^{\mu \nu}(x), \\
\Gamma_{\mu \nu}^{\rho}=\frac{1}{2} \bar{g}^{\rho \sigma}\left(\frac{\partial \bar{g}_{\nu \sigma}}{\partial x^{\mu}}+\frac{\partial \bar{g}_{\mu \sigma}}{\partial x^{v}}-\frac{\partial \bar{g}_{\mu \nu}}{\partial x^{\sigma}}\right), \\
\nabla_{\nu} \bar{\gamma}_{\mu}=\frac{\partial \bar{\gamma}_{\mu}}{\partial x^{\nu}}-\Gamma_{\mu \nu}^{\rho} \bar{\gamma}_{\rho}+\bar{\gamma}_{\mu} \Gamma_{v}-\Gamma_{\nu} \bar{\gamma}_{\mu}=0, \\
\Gamma_{\mu}=-\frac{1}{4} \bar{\gamma}^{\nu}\left(\frac{\partial \bar{\gamma}_{\mu}}{\partial x^{\nu}}-\bar{\gamma}_{\sigma} \Gamma_{\nu \mu}^{\sigma}\right) .
\end{gathered}
$$

The above formula for $\Gamma_{\mu}$ is valid in the case of a diagonal metric $\bar{g}^{\mu v}$ such as the Schwarzschild metric considered in [74]. For a general space-time metric, and with "West-Coast" conventions for the local vierbein $g^{\mu \nu}=\operatorname{diag}(1,-1,-1,-1)$
("East-Coast" conventions were used in [72]), the result reads as

$$
\Gamma_{k}=-\frac{\mathrm{i}}{4} \bar{g}_{\mu \alpha}\left(\frac{\partial b_{v}^{\beta}}{\partial x^{k}} a_{\beta}^{\alpha}-\Gamma_{v k}^{\alpha}\right) \bar{\sigma}^{\mu \nu}
$$

where $\bar{\sigma}^{\mu \nu}=(\mathrm{i} / 2)\left[\bar{\gamma}^{\mu}, \bar{\gamma}^{v}\right]$ is the spin matrix. The $a_{\beta}^{\alpha}$ and $b_{v}^{\beta}$ coefficients transform the Dirac matrices to the local vierbein,

$$
\begin{array}{ll}
\bar{\gamma}_{\rho}=b_{\rho}^{\alpha} \gamma_{\alpha}, & \gamma_{\rho}=a_{\rho}^{\alpha} \bar{\gamma}_{\alpha}, \\
\bar{\gamma}^{\alpha}=a_{\rho}^{\alpha} \gamma^{\rho}, & \gamma^{\alpha}=b_{\rho}^{\alpha} \bar{\gamma}^{\rho} .
\end{array}
$$

With the covariant derivative $\nabla_{\mu}=\partial_{\mu}-\Gamma_{\mu}$, the gravitationally coupled Dirac equation reads as [72-74]

$$
\left(\mathrm{i} \bar{\gamma}^{\mu} \nabla_{\mu}-m\right) \psi(x)=0 .
$$

In the case of a tachyonic Dirac particle, it has to be reformulated as follows:

$$
\left(\mathrm{i} \bar{\gamma}^{\mu} \nabla_{\mu}-\bar{\gamma}^{5}(x) m\right) \psi(x)=0 .
$$

The space-time coordinate-dependent matrix $\bar{\gamma}^{5}(x)$ can be defined as

$$
\begin{aligned}
\bar{\gamma}^{5}(x) & =\frac{\mathrm{i}}{4 !} \frac{\bar{\varepsilon}_{\alpha \beta \gamma \delta}}{\sqrt{-\bar{g}}} \bar{\gamma}^{\alpha} \bar{\gamma}^{\beta} \bar{\gamma}^{\gamma} \bar{\gamma}^{\delta} \\
& =\frac{\mathrm{i}}{4 !} \epsilon_{\alpha \beta \gamma \delta} \bar{\gamma}^{\alpha} \bar{\gamma}^{\beta} \bar{\gamma}^{\gamma} \bar{\gamma}^{\delta}=\mathrm{i} \bar{\gamma}^{0} \bar{\gamma}^{1} \bar{\gamma}^{2} \bar{\gamma}^{3},
\end{aligned}
$$

where $\bar{g}=\operatorname{det} \bar{g}^{\mu v}$ is the determinant of the metric and $\bar{\varepsilon}_{\alpha \beta \gamma \delta}=\sqrt{-\bar{g}} \varepsilon_{\alpha \beta \gamma \delta}$ is the local $\epsilon$ tensor, while $\varepsilon_{\alpha \beta \gamma \delta}$ is the totally antisymmetric Levi-Cività tensor. The last identity in (96) is valid for a diagonal metric $\bar{g}^{\mu \nu}$.

Let us first discuss (94) very briefly. A projection onto the upper and lower radial components $f(r)$ and $g(r)$ in a gravitational field can be found in equations (19) and (20) of [74]. For vanishing electrostatic potential $V \rightarrow 0$, equation (20) of [74] is invariant under the replacement $f(r) \leftrightarrow g(r)$ and $E \leftrightarrow-E$. So, if $E$ is an eigenvalue of the gravitationally coupled Dirac equation, so is $-E$. Invoking reinterpretation and replacing $-E \rightarrow E$ for antiparticles, we find that the spectrum of the gravitationally coupled Dirac Hamiltonian is the same for particles and antiparticles. Therefore, the formalism makes the unique prediction that tardyonic antiparticles, like tardyonic particles, are attracted by a gravitational field. In passing, we note that the often cited motivation for the investigation of trapped antihydrogen and its interaction with the gravitational field therefore is faced with a unique theoretical prediction. Antiparticles are attracted by gravitation as much as particles are.

It has been confirmed within the last two decades [7780] that the Universe expands more rapidly on large distance scales than compatible with the attractive gravitional attraction of the matter density in the Universe. Coupling to a scalar field "quintessence" is usually invoked in order to explain the expansion of the expansion rate of the Universe [81]. As the scalar quintessence field "rolls down its potential," 
it accelerates the expansion rate of the Universe. Because of a self-attracting property (the quintessence field energy can be lowered by increasing the local density of the quintessence field), quintessence has positive energy density but negative pressure. This property is necessary in order to constitute a candidate for dark energy [81]. In the standard model of cosmology, dark energy accounts for about $73 \%$ of the total mass-energy of the Universe. The quintessence field thereby acts like a time-dependent cosmological constant.

We can thus conclude that most of the energy in the Universe actually is not gravitationally attractive; that is, that gravity can repel. In the following, we shall present qualitative arguments which suggest that tachyonic neutrinos may play a role in the expansion of the Universe and may contribute to "dark energy." Conceivable connections of tachyonic physics and dark energy have been explored in the literature, employing either scalar fields [82] or Lorentz-violating mechanisms [83]. In order to provide an alternative explanation for dark energy, it is necessary to invoke a mechanism that leads to a repulsive gravitational force on intergalactic distance scales in the Universe. It is not fully surprising that tachyonic neutrinos may provide for such an alternative mechanism. Namely, both on the classical level [84], and on the level of quantum theory (95), tachyonic particles are repulsed by gravitational fields.

On the classical level, this is seen as follows. We start from the familiar equation of motion of a particle with mass $m$ in curved space-time (see equation (86') of [84]), which is a geodesic,

$$
\frac{\mathrm{d}^{2} x^{\mu}}{\mathrm{d}^{2} s}+\Gamma_{\rho \sigma}^{\mu} \frac{\mathrm{d} x^{\rho}}{\mathrm{d} s} \frac{\mathrm{d} x^{\sigma}}{\mathrm{d} s}=0
$$

With a suitably redefined proper time $\mathrm{d} s^{\prime}$, the zero geodesic for a tachyon reads as (see equation (86') of [84])

$$
\frac{\mathrm{d}^{2} x^{\prime \mu}}{\mathrm{d}^{2} s^{\prime}}+\Gamma_{\rho \sigma}^{\prime \mu} \frac{\mathrm{d} x^{\prime \rho}}{\mathrm{d} s^{\prime}} \frac{\mathrm{d} x^{\prime \sigma}}{\mathrm{d} s^{\prime}}=0 .
$$

The force exerted on a tardyon reads, according to equation (79a) of [84],

$$
F^{\mu}=m \frac{\mathrm{d}^{2} x^{\mu}}{\mathrm{d} s^{2}}=-m \Gamma_{\rho \sigma}^{\mu} \frac{\mathrm{d} x^{\rho}}{\mathrm{d} s} \frac{\mathrm{d} x^{\sigma}}{\mathrm{d} s} .
$$

However, there is a sign change for a tachyon (see equation (79b) of [84]),

$$
F^{\prime \mu}=-m \frac{\mathrm{d}^{2} x^{\prime \mu}}{\mathrm{d} s^{\prime 2}}=m \Gamma_{\rho \sigma}^{\prime \mu} \frac{\mathrm{d} x^{\prime \rho}}{\mathrm{d} s} \frac{\mathrm{d} x^{\prime \sigma}}{\mathrm{d} s}
$$

corresponding to the change in the energy-velocity relation $E=m / \sqrt{1-v^{2}} \rightarrow E=m / \sqrt{v^{2}-1}$ for tardyon versus tachyon, which leads to gravitational repulsion for tachyons. Another intuitive way to understand gravitational tachyonic repulsion is to observe that the quantity $E=\sqrt{\vec{k}^{2}+m^{2}}$ gets smaller when the position-dependent mass $m=m(x)$ gets smaller, whereas the tachyonic energy $E=\sqrt{\vec{k}^{2}-m^{2}}$ gets larger when the position-dependent mass $m=m(x)$ gets smaller.

As neutrinos are ubiquitous within the cosmos, one now needs to evaluate their conceivable contribution to the repulsive force on intergalactic distance scales. A detailed discussion is beyond the scope of the current paper, but we can formulate some initial considerations and order-ofmagnitude estimates. One can identify, a priori, two sources of neutrinos which may become important: (i) thermalized neutrinos which decouple in the early Universe [85-88] and (ii) nonthermalized, high-energy neutrinos which are continuously generated through various cosmic processes, such as nuclear fusion in stars like the sun, supernovae, and high-energy cosmic background.

It is generally assumed that today's cosmic background $(\mathrm{CMB})$ radiation is accompanied by a neutrino background, which at some point (low energy) decoupled from the other particles. The average energy of the background neutrinos is generally assumed to be of the same order-of-magnitude as compared to the cosmic microwave background (CMB), and the currently accepted value for the temperature of the neutrino background is $(4 / 11)^{1 / 3}=0.714$ times that of the electromagnetic background radiation, that is, about $1.9 \mathrm{~K}$. The Universe, according to this hypothesis, would be filled with a sea of nonrelativistic background neutrinos. This picture changes when we assume that the neutrino is tachyonic. In the earliest stages of the Universe, tachyonic neutrinos are of course highly energetic, with $E=$ $\sqrt{\vec{k}^{2}-m^{2}} \approx|\vec{k}| \gg 0$. As they lose energy, they become faster, because $E=m / \sqrt{v^{2}-1} \rightarrow 0$ (in the classical theory). The energy $E=\sqrt{\vec{k}^{2}-m^{2}}$ vanishes as $|\vec{k}| \rightarrow m$, within quantum theory. For $|\vec{k}|<m$, according to [9], the spectrum of the tachyonic Hamiltonian contains unstable resonances and antiresonances, with a purely imaginary resonance energy. Because the real part of the resonance energy $E=-\mathrm{i} \sqrt{m^{2}-\vec{k}^{2}}$ vanishes, the unstable states form a "background" of fluctuating quantum states in the Universe. Particle resonances $\left(E=-\mathrm{i} \sqrt{m^{2}-\vec{k}^{2}}\right)$ and antiparticle antiresonances $\left(E=+i \sqrt{m^{2}-\vec{k}^{2}}\right)$ are damped for propagation into the future and past, respectively (see [9]). The latter case amounts to propagation into the future if one invokes reinterpretation for the antiparticle solutions [9].

A very course-grained estimate regarding the role of the fluctuating resonance and antiresonance states can be given as follows. We start from the grand canonical ensemble

$$
\Omega=-2 \frac{k_{B} T}{2 \pi^{2}} V \int_{0}^{\infty} \mathrm{d} k k^{2} \ln \left(1+\exp \left(\frac{\mu-E(k)}{k_{B} T}\right)\right),
$$

where $k_{B}$ is the Boltzmann constant, $V$ is the normalization volume, $\mu$ is the chemical potential, and $E(k)$ is the energy of a tachyon as a function of $k=|\vec{k}|$. The multiplicity factor 2 takes into account the particle and antiparticle resonances and is supplemented here in comparison to the derivation presented 
in [89]. Furthermore, we have assumed an isotropic energy dependence $\int \mathrm{d}^{3} k \rightarrow 4 \pi \int \mathrm{d} k k^{2}$. The pressure is found as

$$
p=-\frac{\Omega}{V}=\frac{k_{B} T}{\pi^{2}} \int_{0}^{\infty} \mathrm{d} k k^{2} \ln \left(1+\exp \left(\frac{\mu-E(k)}{k_{B} T}\right)\right) .
$$

For $T \rightarrow 0$, the contribution of resonances and antiresonances with $|\vec{k}|<m$ to the pressure and energy density is easily evaluated according to equations (27) and (28) of [89]. For the pressure, we have

$$
\begin{aligned}
p_{0} & =\frac{\mathrm{i}}{3 \pi^{2}} \int_{0}^{m} \mathrm{~d} k k^{3} \frac{\mathrm{d} \operatorname{Im} E(k)}{d k} \\
& =\frac{\mathrm{i}}{3 \pi^{2}} \int_{0}^{m} \mathrm{~d} k \frac{k^{4}}{\sqrt{m^{2}-k^{2}}}=\frac{\mathrm{i} m^{4}}{16 \pi},
\end{aligned}
$$

whereas the energy density is

$$
\begin{aligned}
\rho_{0} & =\frac{\mathrm{i}}{\pi^{2}} \int_{0}^{m} \mathrm{~d} k k^{2} \operatorname{Im} E(k) \\
& =-\frac{\mathrm{i}}{\pi^{2}} \int_{0}^{m} \mathrm{~d} k k^{2} \sqrt{m^{2}-k^{2}}=-\frac{\mathrm{i} m^{4}}{16 \pi}=-p_{0} .
\end{aligned}
$$

The equation of state fulfilled by the zero-temperature limit of the tachyonic Dirac sea is $w_{0}=p_{0} / \rho_{0}=-1$. This is the required equation of state for a "vacuum" energy density that describes a nonvanishing cosmological constant [90]. If $p_{0}+\rho_{0}=0$, then there is no net energy gain upon pulling on a "piston" which contains the Universe (an illustrative discussion can be found in [91]).

Both results in (103) and (104) are imaginary. This raises the pertinent question of how to incorporate the imaginary pressure and energy density of the neutrino field into the evolution equations of the Universe. In giving rise to unstable resonance states, the tachyonic Dirac field, if it exists, would be different from any other known fundamental quantum fields [57]. An exhaustive mathematical description would require an extension of scattering theory to complex energy and momentum exchanges, applied to low-energy collisions with neutrinos, and describe the continuous decay and repopulation of the unstable tachyonic states in the Universe. A detailed discussion is beyond the scope of the current paper. However, in a first approximation, we can argue that an established technique for the mathematical treatment of complex resonances entails complex scaling (for reviews, see $[92,93])$, and we thus explore a complex scaling ansatz to the solution of the evolution equations in the following.

The imaginary results in (103) and (104) describe the quantum fluctuations due to the unstable states. We thus consider equations (8)-(11) of [90], which relate the accelerated expansion rate $\ddot{a} /|a|$ and the Hubble constant $H=\ddot{a} /|a|$ (the dot denotes differentiation with respect to time) to the energy densities of various cosmologically relevant quantities,

$$
H^{2}=\left(\frac{\dot{a}}{|a|}\right)^{2}=\frac{8 \pi G}{3}\left(\rho_{\Lambda}+\rho_{M}+\rho_{k}\right) \approx \frac{8 \pi G}{3}\left(\rho_{\Lambda}+\rho_{M}\right),
$$

$$
\begin{aligned}
\frac{\ddot{a}}{|a|} & =\frac{8 \pi G}{3} \rho_{\Lambda}-\frac{4 \pi G}{3}\left(\rho_{M}+3 p_{M}+\rho_{k}+3 p_{k}\right) \\
& \approx \frac{4 \pi G}{3}\left(2 \rho_{\Lambda}-\rho_{M}\right) .
\end{aligned}
$$

Here, $\rho$ is the matter density in the Universe and $\rho_{k}=$ $-3 k /\left(8 \pi G a^{2}\right)$ is the energy density associated with the curvature of the Universe $(k=+1,0,-1)$, while $\rho_{\Lambda}$ is the energy density corresponding to the cosmological constant. The mass density is $\rho_{M}$ and $p_{M} \approx 0$. We aim to solve (105a) and (105b) using a complex variable ansatz $a=|a| \exp (i \theta)$ and $t=t \exp (i \varphi)$. Using (105a) and (105b), with a value $H=71 \mathrm{~km} \mathrm{~s}^{-1} \mathrm{Mpc}^{-1}$ for the Hubble constant, one easily reproduces the accepted value for the critical mass density of $\rho_{\text {crit }} \approx 9.34 \times 10^{-33} \mathrm{~kg} \mathrm{~cm}^{-3}$ (in SI units) which corresponds to a critical energy density of $\rho_{\text {crit }} \approx 8.39 \times 10^{-9} \mathrm{~J} \mathrm{~m}^{-3}$ (we have set $c=1$ ). In natural units, the result converts to $\rho_{\text {crit }} \approx$ $9.99 \times 10^{-45} \mathrm{GeV}^{4}$.

We courageously set $k=0$ in (105a) and (105b) (flat Universe) and match the modulus of the (complex) accelerated expansion $\ddot{a} /|a|$ against the currently accepted values of $\Omega_{M}=\rho / \rho_{\text {crit }} \approx 0.27$ (see [94]) and $\Omega_{\Lambda}=\rho_{\Lambda} / \rho_{\text {crit }} \approx$ 0.73 . According to (105b), the matching is performed by courageously assuming that the bulk of the cosmological constant is given by the complex energy density (104) and equating the complex modulus as follows:

$$
\begin{gathered}
\rho_{\Lambda}^{\prime} \approx \rho_{0}=-\frac{\mathrm{i} m^{4}}{16 \pi}, \\
\left|2 \rho_{\Lambda}^{\prime}-\rho_{M}\right|=\sqrt{4\left|\rho_{\Lambda}^{\prime}\right|^{2}+\rho_{M}^{2}} \stackrel{!}{=} 2 \rho_{\Lambda}-\rho_{M} \approx 1.19 \rho_{\text {crit }} .
\end{gathered}
$$

The solution is $\rho_{\Lambda}^{\prime}=-\mathrm{i} 0.579 \rho_{\text {crit }}$, and so our estimate for the neutrino mass reads as

$$
\left(5.79 \times 10^{-45} \mathrm{GeV}^{4}\right) \sim\left|\rho_{0}\right|=\frac{m^{4}}{16 \pi} \Longrightarrow m \sim 0.0232 \mathrm{eV} .
$$

If this treatment is valid, then the mass $m$ necessarily has to be the mass of the "heaviest" neutrino mass eigenstate, that is, the one with the largest modulus of the tachyonic mass $m$. Heavier eigenstates would lead to a larger cosmological constant, because $\rho_{0}$ is proportional to the fourth power of the neutrino mass. In terms of a conversion to flavor eigenstates, the heaviest mass eigenstates might well be the one closest to the electron neutrino and electron antineutrino lepton flavor eigenstates [95].

We have interpreted the quantities $p_{0}$ and $\rho_{0}$ given in (103) and (104) as contributions to a cosmological constant which describes the evolution of the Universe in complex space and 
time directions $a=|a| \exp (i \theta)$ and $t=t \exp (\mathrm{i} \varphi)$. We should supplement the solutions for the complex rotation angles for space and time, which read as $\theta=38.1^{\circ}$ and $\varphi=70.6^{\circ}$, within our complex scaling approach. The evolution of the energy density with the aging of the Universe is given by (see equation (12) of [90])

$$
\rho_{0}=\rho_{i}(0) a^{-3\left(1+w_{0}\right)}=\text { const., } \quad w_{0}=-1 .
$$

We therefore obtain a time-independent energy density $\rho_{0}$, which is consistent with the fundamental character of the fluctuating unstable resonances of the neutrino field. We reemphasize that the mass $m \sim 0.0232 \mathrm{eV}$ is tachyonic and enters the gravitationally coupled tachyonic Dirac equation (95). The given mass value is not currently excluded by any terrestrial experiment, and it is consistent with the observed time spread of the arrival times of neutrinos from the supernova SN1987A (see [96]). The hypothesis that very light elementary spin- $1 / 2$ particles could be tachyonic has been pursued elsewhere [97].

\section{Conclusions}

In the current investigation, we present the fundamental solutions of generalized Dirac equations in the helicity basis in a systematic and unified manner. Of particular importance are the Dirac equation with two tardyonic mass terms $m_{1}+$ $\mathrm{i} \gamma^{5} m_{2}$ and two tachyonic mass terms $i m_{1}+\gamma^{5} m_{2}$. Let us summarize the main results. We have discussed the ordinary (tardyonic) Dirac equation in Section 2.2, a tardyonic Dirac equation (with two mass terms) in Section 2.3, and two tachyonic Dirac equations in Sections 3.1 and 3.2. We give the fundamental eigenspinors that enter the plane-wave solutions of all of these equations (see (20a), (20b), (21a), (21b) (27a), (27b), (28a), (28b), (35a), (35b), (36b), (36a), (42a), (42b), (43a) and (43b)). The eigenspinors are obtained using projector techniques as outlined in Chap. 2 of [57]. For the "normal" Dirac equation (see (20a), (20b), (21a), and (21b)), our results are consistent with [3] and Chapter 23 of [58]. For the generalized Dirac equations, the solutions have not yet appeared in the literature in the compact form given in the current paper, to the best of our knowledge. For the ordinary (tardyonic) Dirac equation, for the tachyonic Dirac equation, and for the imaginary-mass Dirac equation, the prefactors are brought into compact analytic form (see (20a), (20b), (21a), (21b), (35a), (35b), (36a), (36b), (42a), (42b), (43a) and (43b)).

Finally, in Sections 4.1 and 4.2, we find that the tardyonic and tachyonic theories can be unified on the basis of the structurally simple anticommutator relations given in (60) and (61), which are independent of the magnitude of the mass terms. As outlined in Section 4.1, the coefficient functions $f=f(\sigma, \vec{k})$ and $g=g(\sigma, \vec{k})$ in the postulated form of the anticommutators (48a) and (48b) enter the tensor sums over the fundamental spinors in (50). For the egregiously simple choices indicated in (60) and (61), which are consistent with a smooth massless limit, the tensor sums over the fundamental eigenspinors yield the positive-energy and negative-energy projectors, for both tardyonic and tachyonic eigenspinors. Consistency with the massless limit requires the presence of the factor $(-\sigma)$ in the tensor sums over the eigenspinors for the tachyonic equations. This fact is verified, on the basis of tachyonic Gordon identities and related considerations, in Sections 4.3 and 4.4. The presence of the factor $(-\sigma)$ in the fundamental tachyonic field anticommutators in (61) implies the suppression of right-handed particle and lefthanded antiparticle states, due to negative norm, as shown in (72).

Finally, in Section 5, we observe that since tachyons are repelled by gravity, it might be worthwhile to investigate their conceivable role in the mechanism(s) responsible for the accelerated expansion of the Universe "dark energy." Furthermore, the tachyonic resonances and antiresonance energies might play a role in the sum over states that enter the thermodynamic potentials of a free tachyonic fermionic gas in the low-temperature limit. If we consider the states with imaginary energy to be unstable, fluctuating states, then it is intuitively obvious that they might contribute to a fluctuating energy density and pressure on large distance scales in the Universe. As described in an approach in Section 5.3, an order-of-magnitude calculation based on a complex scaling transformation of the time evolution of the Universe leads to a tachyonic neutrino mass which is not excluded at present by any terrestrial experiment. We believe that it might be worthwhile to explore the physical consequences of the "ugly duckling" (tachyonic neutrino) somewhat further. It allows us to retain, among other things, lepton number conservation as a symmetry of nature.

\section{Acknowledgments}

The authors acknowledge helpful conversations with C. Hirata, R. J. Hill, W. Rodejohann, P. J. Mohr, and I. Nándori. This work was supported by the NSF (Grant PHY-1068547) and by the National Institute of Standards and Technology (Precision Measurement Grant).

\section{References}

[1] P. A. M. Dirac, "The quantum theory of the electron," Proceedings of the Royal Society A, vol. 117, pp. 610-624, 1928.

[2] P. A. M. Dirac, "The quantum theory of the electron," Proceedings of the Royal Society A, vol. 118, pp. 351-361, 1928.

[3] M. Jacob and G. C. Wick, "On the general theory of collisions for particles with spin," Annals of Physics, vol. 7, pp. 404-428, 1959.

[4] M. Joyce, K. Kainulainen, and T. Prokopec, "Fermion propagator in a non-trivial background field," Journal of High Energy Physics, vol. 4, no. 10, article 029, 2000.

[5] P. Mastrolia, private communication, 2012.

[6] A. Chodos, A. I. Hauser, and V. A. Kostelecký, "The neutrino as a tachyon," Physics Letters B, vol. 150, no. 6, pp. 431-435, 1985.

[7] T. Chang, "A new Dirac-type equation for tachyonic neutrinos," http://arxiv.org/abs/hep-th/0011087.

[8] T. Chang, "Parity violation and neutrino mass," http://arxiv.org/ abs/hep-ph/0208239.

[9] U. D. Jentschura and B. J. Wundt, "Localizability of tachyonic particles and neutrinoless double beta decay," The European Physical Journal C, vol. 72, p. 1894, 2012. 
[10] U. D. Jentschura and B. J. Wundt, "Pseudo-Hermitian quantum dynamics of tachyonic spin-1/2 particles," Journal of Physics A, vol. 45, no. 44, Article ID 444017, 2012.

[11] G. Akemann, P. H. Damgaard, K. Splittor, and J. Verbaarschoot, "Wilson Fermions, random matrix theory and the Aoki phase," in Proceedings of the 28th International Symposium on Lattice Field Theory (Lattice '10), Villasimius, Italy, June 2010, http://arxiv.org/abs/1011.5118.

[12] C. Gattringer and C. B. Lang, Quantum Chromodynamics on the Lattice: An Introductory Presentation, vol. 788 of Lecture Notes in Physics, Springer, Berlin, Germany, 2010.

[13] W. Pauli, "On Dirac's new method of field quantization," Reviews of Modern Physics, vol. 15, pp. 175-207, 1943.

[14] C. M. Bender and S. Boettcher, "Real spectra in non-Hermitian Hamiltonians having $\mathscr{P} \mathscr{T}$ symmetry," Physical Review Letters, vol. 80, no. 24, pp. 5243-5246, 1998.

[15] C. M. Bender and G. V. Dunne, "Large-order perturbation theory for a non-Hermitian $\mathscr{P} \mathscr{T}$-symmetric Hamiltonian," Journal of Mathematical Physics, vol. 40, no. 10, pp. 4616-4621, 1999.

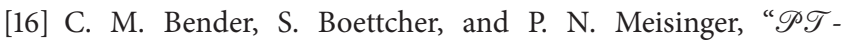
symmetric quantum mechanics," Journal of Mathematical Physics, vol. 40, no. 5, pp. 2201-2229, 1999.

[17] C. M. Bender and E. J. Weniger, "Numerical evidence that the perturbation expansion for a non-Hermitian $\mathscr{P} \mathscr{T}$-symmetric Hamiltonian is Stieltjes," Journal of Mathematical Physics, vol. 42, no. 5, pp. 2167-2183, 2001.

[18] C. M. Bender, D. C. Brody, and H. F. Jones, "Complex extension of quantum mechanics," Physical Review Letters, vol. 89, no. 27, Article ID 270401, 4 pages, 2002.

[19] A. Mostafazadeh, "Pseudo-Hermiticity versus $\mathscr{P} \mathscr{T}$ symmetry: the necessary condition for the reality of the spectrum of a nonHermitian Hamiltonian," Journal of Mathematical Physics, vol. 43, no. 1, pp. 205-214, 2002.

[20] A. Mostafazadeh, "Pseudo-Hermiticity versus $\mathscr{P} \mathscr{T}$-symmetry. II: a complete characterization of non-Hermitian Hamiltonians with a real spectrum," Journal of Mathematical Physics, vol. 43, no. 5, pp. 2814-2816, 2002.

[21] A. Mostafazadeh, "Pseudo-Hermiticity and generalized PTand CPT-symmetries," Journal of Mathematical Physics, vol. 44, no. 3, pp. 974-989, 2003.

[22] U. D. Jentschura, A. Surzhykov, and J. Zinn-Justin, "Unified treatment of even and odd anharmonic oscillators of arbitrary degree," Physical Review Letters, vol. 102, no. 1, Article ID 011601, 2009.

[23] U. D. Jentschura, A. Surzhykov, and J. Zinn-Justin, "Multiinstantons and exact results III: unification of even and odd anharmonic oscillators," Annals of Physics, vol. 325, no. 5, pp. 1135-1172, 2010.

[24] J. Bandukwala and D. Shay, "Theory of free, spin-1/2 tachyons," Physical Review D, vol. 9, no. 4, pp. 889-895, 1974.

[25] U. D. Jentschura, "Dirac Hamiltonian with imaginary mass and induced helicity-dependence by indefnite metric," Journal of Modern Physics, vol. 3, no. 9, pp. 887-894, 2012.

[26] A. Szameit, M. C. Rechtsman, O. Bahat-Treidel, and S. Mordechai, "PT-symmetry in honeycomb photonic lattices," Physical Review A, vol. 84, no. 2, Article ID 021806(R), 2012.

[27] G. Székely, "The existence of superluminal particles is consistent with the kinematics of Einstein's special theory of relativity," http://arxiv.org/abs/1202.5790.
[28] O. M. P. Bilaniuk, V. K. Deshpande, and E. C. G. Sudarshan, “"Meta' relativity," American Journal of Physics, vol. 30, no. 10, pp. 718-723, 1962.

[29] M. E. Arons and E. C. G. Sudarshan, "Lorentz invariance, local field theory, and faster-than-light particles," Physical Review, vol. 173, no. 5, pp. 1622-1628, 1968.

[30] J. Dhar and E. C. G. Saudarshan, "Quantum field theory of interacting tachyons," Physical Review, vol. 174, no. 5, pp. 1808$1815,1968$.

[31] O. M. Bilaniuk and E. C. G. Sudarshan, "Causality and spacelike signals," Nature, vol. 223, no. 5204, pp. 386-387, 1969.

[32] G. Feinberg, "Possibility of faster-than-light particles," Physical Review, vol. 159, no. 5, pp. 1089-1105, 1967.

[33] G. Feinberg, "Lorentz invariance of tachyon theories," Physical Review D, vol. 17, no. 6, pp. 1651-1660, 1978.

[34] U. D. Jentschura, "Tachyonic field theory and neutrino mass running," Central European Journal of Physics, vol. 10, no. 4, pp. 749-762, 2012.

[35] R. Ehrlich, "Implications for the cosmic ray spectrum of a negative electron neutrino (mass) ${ }^{2}$," Physical Review D, vol. 60, no. 1, Article ID 017302, 4 pages, 1999.

[36] R. Ehrlich, "Is there a $4.5 \mathrm{PeV}$ neutron line in the cosmic ray spectrum?” Physical Review D, vol. 60, no. 7, Article ID 073005, 1999.

[37] R. Ehrlich, "Neutrino mass ${ }^{2}$ inferred from the cosmic ray spectrum and tritium beta decay," Physics Letters B, vol. 493, no. 3-4, pp. 229-232, 2000.

[38] R. Ehrlich, "Evidence for two neutrino mass eigenstates from SN 1987A and the possibility of superluminal neutrinos," Astroparticle Physics, vol. 35, pp. 625-628, 2012.

[39] R. Ehrlich, "The superluminal neutrino hypothesis and the neutrino mass hierarchy," Astroparticle Physics, vol. 41, pp. 1-6, 2013.

[40] G. R. Kalbfleisch, N. Baggett, E. C. Fowler, and J. Alspector, "Experimental comparison of neutrino, antineutrino, and muon velocities," Physical Review Letters, vol. 43, no. 19, pp. 1361-1364, 1979.

[41] P. Adamson, C. Andreopoulos, K. E. Arms et al., "Measurement of neutrino velocity with the MINOS detectors and NuMI neutrino beam," Physical Review D, vol. 76, no. 7, Article ID 072005, 6 pages, 2007.

[42] T. Adam, N. Agafonova, A. Aleksandrov et al., "Measurement of the neutrino velocity with the OPERA detector in the CNGS beam," Journal of High Energy Physics, vol. 2012, no. 10, article 93, 2012.

[43] M. Antonello, P. Aprili, B. Baiboussinov et al., "Measurement of the neutrino velocity with the ICARUS detector at the CNGS beam," Physics Letters B, vol. 713, no. 1, pp. 17-22, 2012.

[44] R. G. H. Robertson, T. J. Bowles, G. J. Stephenson, D. L. Wark, J. F. Wilkerson, and D. A. Knapp, "Limit on $\bar{v}_{e}$ mass from observation of the $\beta$ decay of molecular tritium," Physical Review Letters, vol. 67, no. 8, pp. 957-960, 1991.

[45] K. Assamagan, C. Brönnimann, M. Daum et al., "Measurement of the muon momentum in pion decay at rest using a surface muon beam," Physics Letters B, vol. 335, no. 2, pp. 231-236, 1994.

[46] W. Stoeffl and D. J. Decman, "Anomalous structure in the beta decay of gaseous molecular tritium," Physical Review Letters, vol. 75, no. 18, pp. 3237-3240, 1995.

[47] K. Assamagan, C. Brönnimann, M. Daum et al., "Upper limit of the muon-neutrino mass and charged-pion mass from 
momentum analysis of a surface muon beam," Physical Review D, vol. 53, no. 11, pp. 6065-6077, 1996.

[48] C. Weinheimer, B. Degenddag, A. Bleile et al., "High precision measurement of the tritium $\beta$ spectrum near its endpoint and upper limit on the neutrino mass," Physics Letters B, vol. 460, no. 1-2, pp. 219-226, 1999.

[49] V. M. Lobashev, V. N. Aseev, A. I. Belesev et al., "Direct search for mass of neutrino and anomaly in the tritium beta-spectrum," Physics Letters B, vol. 460, no. 1-2, pp. 227-235, 1999.

[50] A. I. Belesev, E. V. Geraskin, B. L. Zhuikov et al., "Investigation of space-charge effects in gaseous tritium as a source of distortions of the beta spectrum observed in the Troitsk neutrinomass experiment," Physics of Atomic Nuclei, vol. 71, no. 3, pp. 427-436, 2008.

[51] M. Dracos, "The neutrino velocity measurement by OPERA experiment," in Proceedings of the 25th International Conference on Neutrino Physics and Astrophysics, pp. 1-15, Kyoto, Japan, June 2012, http://regmedia.co.uk/2012/06/11/neutrinos_not_ $\mathrm{ftl}$ slides.pdf.

[52] U. D. Jentschura and B. J. Wundt, "An infinitesimally superluminal Neutrino is left-handed, conserves Lepton number and solves the Autobahn Paradox (Illustrative Discussion)," http://arxiv.org/abs/1206.6342.

[53] W. Pauli, "Letter to a physicists gathering at Tübingen, December 4, 1930," in Collected Scientific Papers, R. Kronig and V. Weisskopf, Eds., vol. 2, p. 1313, Interscience, New York, NY, USA, 1964.

[54] F. Reines and C. L. Cowan, “The neutrino," Nature, vol. 178, p. 446, 1956.

[55] C. Enz and K. von Meyenn, Wolfgang Pauli, a Biographical Introduction, Springer, Heidelberg, Germany, 1994.

[56] V. A. Kostelecky and M. Mewes, "Neutrinos with Lorentzviolating operators of arbitrary dimension," Physical Review D, vol. 85, no. 9, Article ID 096005, 36 pages, 2012.

[57] C. Itzykson and J. B. Zuber, Quantum Field Theory, McGrawHill, New York, NY, USA, 1980.

[58] V. B. Berestetskii, E. M. Lifshitz, and L. P. Pitaevskii, Quantum Electrodynamics, Pergamon Press, Oxford, UK, 2nd edition, 1982.

[59] M. Pavsic, "Localized propagating tachyons in extended relativity theories," http://arxiv.org/abs/1201.5755.

[60] M. E. Peskin and D. V. Schroeder, An Introduction to Quantum Field Theory, Perseus, Cambridge, Mass, USA, 1995.

[61] S. S. Schweber, An Introduction to Relativistic Quantum Field Theory, Harper \& Row, New York, NY, USA, 1961.

[62] R. J. Hughes and G. J. Stephenson Jr., "Against tachyonic neutrinos," Physics Letters B, vol. 244, no. 1, pp. 95-100, 1990.

[63] C. M. Bender, J. Brod, A. Refig, and M. E. Reuter, "The $\mathscr{C}$ operator in $\mathscr{P} \mathscr{T}$-symmetric quantum theories," Journal of Physics A, vol. 37, pp. 10139-10165, 2004.

[64] C. M. Bender, S. F. Brandt, J. H. Chen, and Q. Wang, "The $\mathscr{C}$ operator in $\mathscr{P} \mathscr{T}$-symmetric quantum field theory transforms as a Lorentz scalar," Physical Review D, vol. 71, no. 6, Article ID 065010, 7 pages, 2005.

[65] Y. Aharonov, B. Reznik, and A. Stern, "Quantum limitations on superluminal proagation," Physical Review Letters, vol. 81, no. 11, pp. 2190-2193, 1998.

[66] A. Enders and G. Nimtz, "On superluminal barrier traversal," Journal de Physique I, vol. 2, no. 2, pp. 1693-1698, 1992.

[67] G. Nimtz and A. A. Stahlhofen, "Universal tunneling time for all fields," Annalen der Physik, vol. 17, no. 6, pp. 374-379, 2008.
[68] G. Nimtz, "On virtual phonons, photons and electrons," Foundations of Physics, vol. 39, p. 1346, 2009.

[69] S. M. Bilenky and C. Giunti, "Neutrinoless double-beta decay: a brief review," Modern Physics Letters A, vol. 27, no. 13, Article ID 1230015, 22 pages, 2012.

[70] E. Trojan, "Interacting tachyon Fermi gas," http://arxiv.org/abs/ 1203.5241.

[71] H. V. Klapdor-Kleingrothaus, I. V. Krivosheina, A. Dietz, and O. Chkvorets, "Search for neutrinoless double beta decay with enriched ${ }^{76}$ Ge in Gran Sasso 1990-2003," Physics Letters B, vol. 586, no. 3-4, pp. 198-212, 2004.

[72] D. R. Brill and J. A. Wheeler, "Interaction of neutrinos and gravitational fields," Reviews of Modern Physics, vol. 29, no. 3, pp. 465-479, 1957.

[73] D. G. Boulware, "Spin-1/2 quantum field theory in Schwarzschild space," Physical Review D, vol. 12, no. 2, pp. 350-367, 1975.

[74] M. Soffel, B. Müller, and W. Greiner, "Particles in a stationary spherically symmetric gravitational field," Journal of Physics A, vol. 10, p. 551, 1977.

[75] G. V. Shishkin and W. D. Cabos, "Dirac equation in external fields: separation of variables in curvilinear coordinates," Journal of Mathematical Physics, vol. 33, no. 3, pp. 914-925, 1992.

[76] G. V. Shishkin and V. M. Villalba, "Neutrino in the presence of gravitational fields: exact solutions," Journal of Mathematical Physics, vol. 33, no. 12, pp. 4037-4045, 1992.

[77] G. Efstathiou, W. J. Sutherland, and S. J. Maddox, "The cosmological constant and cold dark matter," Nature, vol. 348, no. 6303, pp. 705-707, 1990.

[78] J. Hoell and W. Priester, "Dark matter and the cosmological constant," Comments on Astrophysics, vol. 15, no. 3, pp. 127-138, 1991.

[79] S. Perlmutter, "Cosmology from type Ia supernovae," Bulletin of the American Astronomical Society, vol. 29, p. 1351, 1998.

[80] A. G. Riess, A. V. Filippenko, P. Challis et al., "Observational evidence from supernovae for an accelerating universe and a cosmological constant," Astronomical Journal, vol. 116, no. 3, pp. 1009-1038, 1998.

[81] P. J. Steinhardt, "A quintessential introduction to dark energy," Philosophical Transactions of the Royal Society A, vol. 361, no. 1, pp. 2497-2513, 2003.

[82] J. S. Bagla, H. K. Jassal, and T. Padmanabhan, "Cosmology with tachyon field as dark energy," Physical Review D, vol. 67, no. 6, Article ID 063504, 11 pages, 2003.

[83] E. Ciuoli, J. Evslin, J. Liu, and X. Zhang, "Neutrino dispersion relations from a dark energy model," http://arxiv.org/abs/ 1109.6641.

[84] E. Recami and R. Mignani, "Classical theory of tachyons (special relativity extended to superluminal frames and objects)," $L a$ Rivista del Nuovo Cimento Series 2, vol. 4, no. 2, pp. 209-290, 1974.

[85] S. Hannestad and J. Madsen, "Neutrino decoupling in the early Universe," Physical Review D, vol. 52, no. 4, pp. 1764-1769, 1995.

[86] R. E. Lopez, S. Dodelson, A. Heckler, and M. S. Turner, "Precision detection of the cosmic neutrino background," Physical Review Letters, vol. 82, no. 20, pp. 3952-3955, 1999.

[87] R. Trotta and A. Melchiorri, "Indication for primordial anisotropies in the neutrino background from WMAP and SDSS," Physical Review Letters, vol. 95, Article ID 011305, 2005. 
[88] S. Hannestad, "New CMBR data and the cosmic neutrino background," Physical Review D, vol. 64, no. 8, Article ID 083002, 2001.

[89] E. Trojan, “Tachyonic Dirac sea," http://arxiv.org/abs/1201.6560.

[90] http://www.scholarpedia.org/article/Cosmological_constant\# Equivalence_of_Cosmological_Constant_and_Vacuum_Energy.

[91] http://www.astro.ucla.edu/ wright/cosmo_constant.html.

[92] N. Moiseyev, "Quantum theory of resonances: calculating energies, widths and cross-sections by complex scaling," Physics Reports, vol. 302, no. 5-6, pp. 211-293, 1998.

[93] E. Caliceti, M. Meyer-Hermann, P. Ribeca, A. Surzhykov, and U. D. Jentschura, "From useful algorithms for slowly convergent series to physical predictions based on divergent perturbative expansions," Physics Reports, vol. 446, no. 1-3, pp. 1-96, 2007.

[94] M. Donahue and G. M. Voit, " $\Omega_{m}$ from the temperature-redshift distribution of EMSS clusters of galaxies," Astrophysical Journal Letters, vol. 523, no. 2, pp. L137-L140, 1999.

[95] S. M. Bilenky, "Majorana neutrino mixing," Journal of Physics G, vol. 32, no. 5, pp. R127-R149, 2006.

[96] W. D. Arnett and J. L. Rosner, "Neutrino mass limits from SN1987A," Physical Review Letters, vol. 58, no. 18, pp. 1906-1909, 1987.

[97] D. V. Ahluwalia-Khalilova and I. Dymnikova, "A theoretical case for negative mass-square for sub-eV particles," International Journal of Modern Physics D, vol. 12, no. 9, pp. 1787-1794, 2003. 

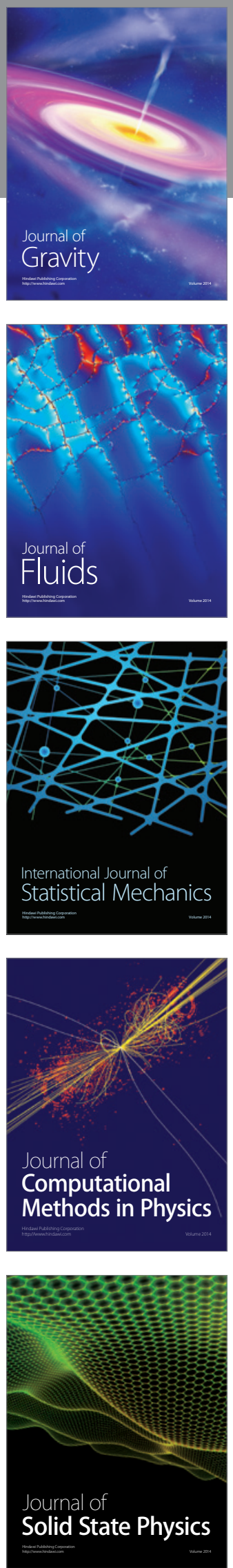

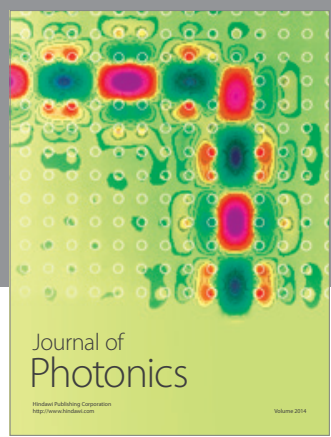

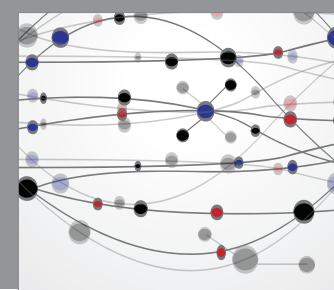

The Scientific World Journal

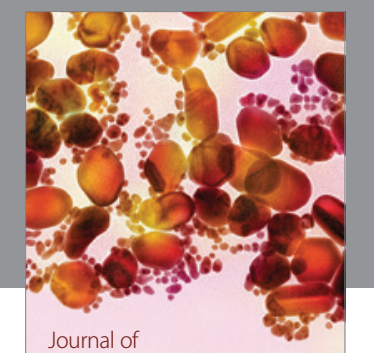

Soft Matter
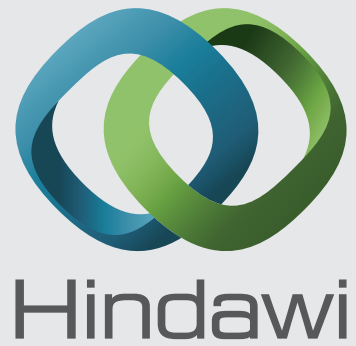

Submit your manuscripts at

http://www.hindawi.com
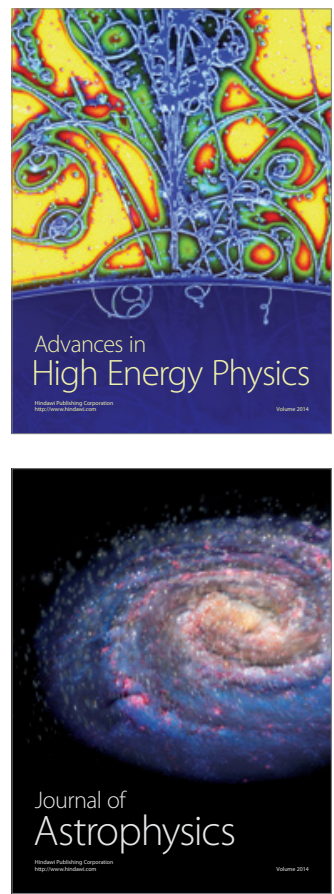
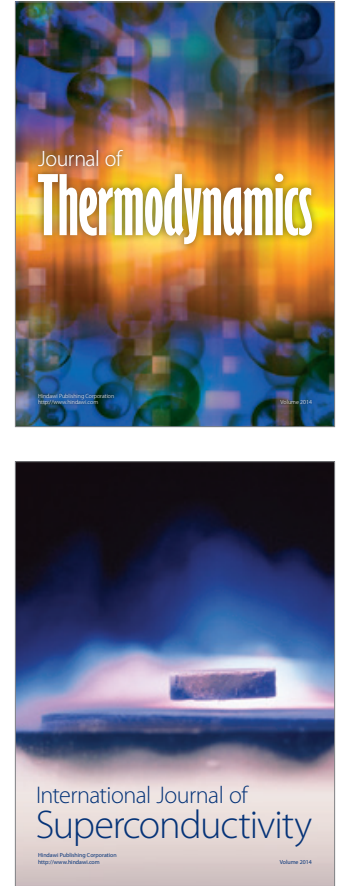
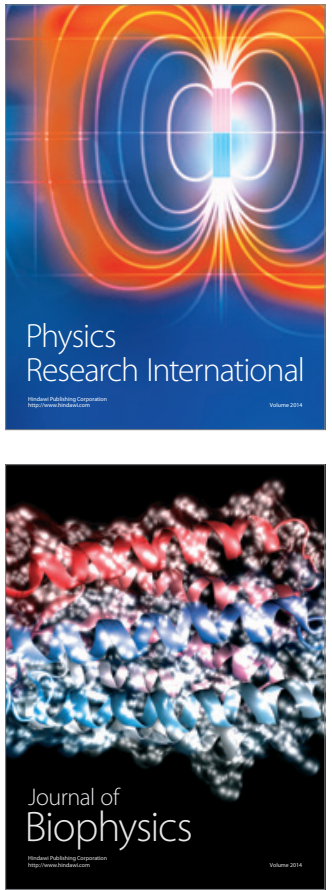
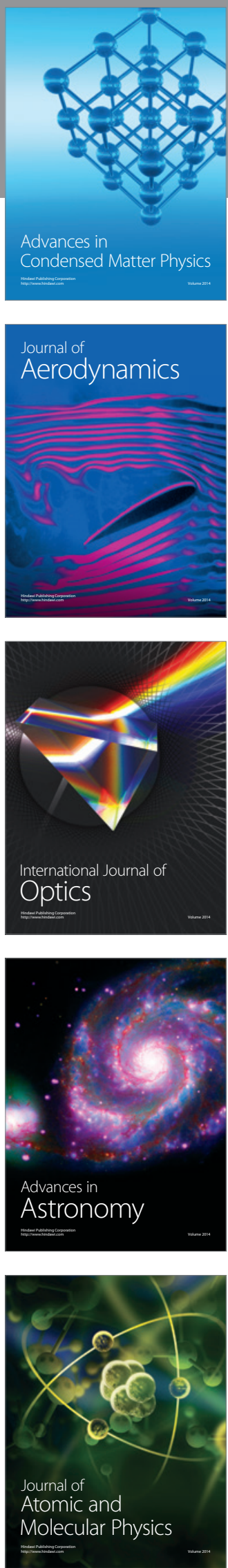